\title{
Osteoblast lineage-specific cell-surface antigen (A7) regulates osteoclast recruitment and calcification during bone remodeling
}

\author{
Tamer Badawy ${ }^{1,3} \cdot$ Yukari Kyumoto-Nakamura ${ }^{1} \cdot$ Norihisa Uehara $^{1} \cdot$ Jingqi Zhang $\mathbb{D}^{1} \cdot$ Soichiro Sonoda $^{1} \cdot$ \\ Hidenobu Hiura ${ }^{1} \cdot$ Takayoshi Yamaza $\mathbb{D}^{1} \cdot$ Akiko Kukita $\mathbb{D}^{2} \cdot$ Toshio Kukita $^{1}$
}

Received: 20 April 2018 / Revised: 19 October 2018 / Accepted: 13 November 2018 / Published online: 11 February 2019

(c) United States \& Canadian Academy of Pathology 2019

\begin{abstract}
Bone remodeling is a continuous process characterized by highly coordinated cell-cell interactions in distinct multi-cellular units. Osteoclasts, which are specialized bone resorbing cells, play a central role in bone remodeling. Although the RANKL/ RANK axis determines the gross number of osteoclasts present in bone tissue, detailed molecular events regulating bone remodeling related to osteoclast recruitment, initiation of bone remodeling, and coupling of bone resorption and bone formation are still ambiguous. We hypothesized that osteoblast-specific cell-surface molecules contribute to the molecular modulation of bone remodeling. Therefore, we searched for regulatory cell-surface molecules expressed on osteoblasts by use of B-cell hybridoma technology. We obtained a monoclonal antibody A7 (A7 MAb) highly specific to cells of osteoblast-lineage. Here we describe the expression pattern and possible role of A7 antigen specifically recognized by A7 MAb. In vitro, A7 antigen was expressed on cell-surface of osteoblasts and osteoblast-like bone marrow stromal cells. In vivo, A7 antigen was detected in a subset of bone surface osteoblasts and in osteocytes, with a typical cell membrane expression pattern. Tissue array analysis showed only a limited expression of A7 antigen in osteocytes close to the bone surface. Immunoblotting and immunoprecipitation analysis showed that A7 antigen is a lineage-specific cell-surface protein with an approximate molecular weight of $45 \mathrm{KDa}$. Cross-linking of cell-surface A7 antigen in cultures of osteoclastogenesis showed stimulation of osteoclast formation. Marked suppression of calcification in primary osteoblast cultures was observed when A7 antigen was cross-linked with anti-A7 antigen MAb, A7 MAb. These data suggest that A7 antigen regulates recruitment of osteoclasts and triggering of calcification. A7 antigen may be an important molecule involved in the precise regulation of bone remodeling.
\end{abstract}

Supplementary information The online version of this article (https:// doi.org/10.1038/s41374-018-0179-4) contains supplementary material, which is available to authorized users.

$\triangle$ Toshio Kukita

kukitat@dent.kyushu-u.ac.jp

1 Department of Molecular Cell Biology and Oral Anatomy, Faculty of Dental Science, Kyushu University, Maidashi, Fukuoka 8128582, Japan

2 Department of Microbiology, Faculty of Medicine, Saga University, Nabeshima, Saga 894-8501, Japan

3 Department of Oral Biology, Faculty of Dentistry, Cairo University, Cairo 11553, Egypt

\section{Introduction}

Bone is a highly dynamic tissue undergoing constant remodeling and turnover throughout life. Bone remodeling consists of cycles of bone resorption coupled with subsequent bone formation mediated by osteoblasts [1]. Osteoclasts, which are bone resorbing cells, play a central role in bone remodeling [2]. In normal, healthy bone, remodeling takes place at specified discrete sites known as basic multicellular units or bone remodeling compartments (BRC) [3]. At each BRC, remodeling occurs in sequential complex phases. In the initial phase, unknown triggering signals are induced on the cell surfacse of osteoblasts and/or bone lining cells, providing the site of bone remodeling onset, where osteoclasts are recruited. In the following resorption phase, bone resorption predominates together with induction of differentiation of mesenchymal stem cells and osteoprogenitor cells into osteoblasts. Next is 
the reversal phase, in which osteoclasts stop bone resorption and bone matrix is deposited by newly formed osteoblasts, followed by subsequent mineralization in the final phase $[4,5]$.

In pathological bone destruction, significant abnormalities are often observed in bone remodeling, in which aggressive bone resorption occurs without adequate supplementation of bone [6-8]. One possibility is that the number of initiation sites of bone remodeling dramatically increases, so that bone formation is not able to reach equilibrium with the required apposition of new bone. The other possibility is that modified osteoclasts are present in the area of pathological bone destruction. We have recently proposed a hypothesis that pathologically activated osteoclasts constitute a unique subpopulation that differs from normal osteoclasts and bears aggressive bone resorbing activity [9]. In both cases, osteoclast recruitment at specified areas of bone surface triggers the initiation of bone remodeling as well as pathological bone destruction.

As osteoclasts are formed and recruited on bone lining cells or on osteoblasts that cover bone matrices, it is reasonable to postulate that cell-surface molecules expressed on cells of osteoblast-lineage have pivotal regulatory roles in the recruitment of osteoclasts at sites of bone remodeling either under normal conditions or in pathological environments. Receptor activator of NF- $k$ B ligand (RANKL) is an essential osteoclast differentiation factor expressed on the cell surface of osteoblasts [10-12]. Osteoblasts also produce osteoprotegerin (OPG), which neutralizes the action of RANKL to inhibit osteoclastogenesis [13]. Formation of osteoclasts are believed to be determined by the ratio of RANKL/OPG [14]. However, the molecular regulation of coupling between osteoclasts and osteoblasts is likely a more complex process in which other potent regulatory molecules are involved.

On the other hand, new bone formation at each resorption lacuna formed by osteoclasts is essential for the completion of one cycle of bone remodeling. Ephrin B2 ligands and their receptor EphB4 are mainly expressed on cell surface of endothelial cells of arteries and veins, respectively and act as key molecules in the formation of blood vessel networks [15]. At the transition phase of bone remodeling, cell-cell interactions between osteoblasts and osteoclasts are mediated by surface ephrin B2 expressed on osteoclasts and their receptors EphB4 expressed on cell surfaces of osteoblasts [16]. Interaction of ephrin B2 and EphB4 results in the stimulation of osteoblast formation and suppression of osteoclastogenesis [16]. The ephrin B2/Eph B4 system seems to be important in the transition stage of bone remodeling, however, this system is widely utilized to the formation of blood vessel network, bone might have a more specific regulatory system for osteoblast maturation involving calcification.
In order to determine the precise mechanisms of modulation of bone remodeling, i.e., specific events in bone tissue, it is valuable to identify cell surface molecules specifically expressed on cells of osteoblast-lineage covering the bone matrices. To investigate cell surface molecules which regulate initial osteoclast recruitment and final maturation of osteoblasts to form calcified bone matrices, we developed a panel of monoclonal antibodies that recognize cell surface of osteoblasts. Here we report on a unique cell-surface antigen specifically expressed in cells of osteoblast-lineage. Cross-linking of the cell surface antigen by using the specific monoclonal antibody show that this osteoblast-specific cell surface antigen is not only involved in the modulation of osteoclastogenesis but also has pivotal regulatory roles in the calcification mediated by mature osteoblasts. This osteoblast-specific cell surface molecule may be a key player in the precise regulation of bone remodeling in healthy conditions as well as in pathological environments.

\section{Materials and methods}

\section{Animals}

Female BALB/cN Sea mice were purchased from Kyudo (Tosu, Japan) and were used for antigen injection for hybridoma formation. Male Sprague-Dawley (SD) rats were purchased from CLEA (Tokyo, Japan) and were used for whole rat bone marrow culture for osteoclastogenesis. Female BALB/cCr mice, female $\mathrm{LEW} / \mathrm{SsN}$ rats and neonatal SD rats were purchased from SLC (Fukuoka, Japan). All animal experiments were performed according to the guidelines for care and use of animals of Kyushu University.

\section{Cell lines and culture}

The rat osteosarcoma cell line ROS17/2.8 was kindly provided by Dr. David Roodman (Indiana University School of Medicine). Human osteosarcoma cell line MG-63 and Saos2 were provided by Dr. Hirata (Kyushu University, School of Medicine). Rat osteoblastic cell line UMR106 and rat myoblast cell line L8 and rat alveolar macrophage cell line NR8383 were obtained from Riken cell bank (Tsukuba, Japan). Murine osteoblast cell line MC-3T3E1 was kindly provided by Dr. Jimi and Dr. Matsuda of Kyushu University, Faculty of Dental Science. MG-63, Saos-2, ROS17/ 2.8, UMR106 and L8 cells were cultured in DMEM (Gibco, Grand Island, NY, USA) containing $10 \%$ fetal bovine serum (FBS) (Life Technologies, Grand Island, NY, USA). NR8383 cells were cultured in F-12K HAM (ATCC, Manassas, VA, USA) containing $15 \%$ FBS. Osteoclast 
precursor cell line RAW-D was cultured in $\alpha$-MEM, $10 \%$ FBS in presence or absence of RANKL $(50 \mathrm{ng} / \mathrm{ml})[17,18]$. MC-3T3E1 cells were cultured in $\alpha$-MEM with L-glutamine, $10 \%$ FBS. Cell lines were cultured in $60-\mathrm{mm}$ or $100-$ mm (Becton Dickinson, Franklin Lakes, NJ, USA) tissue culture dishes and in 96-multiwell plates. $\alpha$-MEM with Lglutamine and phenol red was purchased from WAKO (Tokyo, Japan). For cell passaging, $0.05 \%$ Trypsin $/ 0.02 \%$ EDTA solution was used for all cell lines except for NR8383 and RAW-D cell lines.

\section{Immunocytochemical staining of unfixed culture cells, Immunization and Hybridoma formation}

After cells reached sub-confluency, unfixed cells were reacted with primary antibodies at $4{ }^{\circ} \mathrm{C}$ for $30 \mathrm{~min}$. The cells were then rinsed in serum-free $\alpha$-MEM or DMEM 4 times, fixed in $4 \%$ paraformaldehyde in PBS (pH 7.4) at room temperature for 20 min, and rinsed in PBS five times. After blocking with $3 \%$ goat serum in PBS for $30 \mathrm{~min}$, cells were reacted with biotinylated anti-mouse $\operatorname{IgG}(\mathrm{H}+\mathrm{L})$ (Vector Laboratories, Burlingame, CA, USA) in $1 \%$ horse serum in PBS at room temperature for $30 \mathrm{~min}$. The cells were further reacted with ABC-AP complex prepared by use of Vectastain ${ }^{\circ} \mathrm{ABC}-\mathrm{AP}$ staining kit (Alkaline Phosphatase Standard, AK-5000) (Vector Laboratories) in 1\% horse serum in PBS at room temperature for $30 \mathrm{~min}$. Color development was performed by use of Vector ${ }^{\oplus}$ Red Alkaline Phosphatase substrate kit SK-5100 (Vector Laboratories, Burlingame, CA, USA) in the presence of $1 \mathrm{mM}$ levamisole according to the manufacturer's protocol. After color development, the cells were fixed in 5\% glutaraldehyde in PBS for $30 \mathrm{~min}$ at room temperature, rinsed in distilled water 4 times, and then air dried. Immunization and Hybridoma formation ROS17/2.8 cell clone was obtained from original ROS17/2.8 rat osteosarcoma cell line [19] using the standard limiting dilution method. The rapid proliferating ROS17/2.8 cell clone with the typical polygonal morphology of osteoblasts was termed "ROS17/2.8 CL\#7" and was used as the immunogen for mice immunization. ROS17/2.8 CL\#7 was cultured in 100$\mathrm{mm}$ culture dishes and after reaching $80 \%$ confluency, cells were washed twice in phosphate buffered saline (PBS) and trypsinized using $0.05 \%$ Trypsin, $0.02 \%$ EDTA. Single cell suspension was obtained after cells were collected and centrifuged at $1000 \mathrm{rpm}$ for $10 \mathrm{~min}$ at $4{ }^{\circ} \mathrm{C}$. Cells were injected intraperitoneally (i.p.) into isoflurane-anesthetized female $\mathrm{BALB} / \mathrm{cN}$ Sea mice using 26 -gauge needle $\left(1 \times 10^{7}\right.$ cells $/ 500$ $\mu \mathrm{l}$ of ice-chilled PBS per mouse). Mice were then boosted two times every two weeks. Mice were then sacrificed 3 days after the second boost for hybridoma production. Formation of Bcell hybridomas was performed basically according to the method of Köhler and Milstein (1975) [20] as modified by Reading (1982) [21], where the spleen cells obtained from the immunized mice were fused with murine myeloma cell line
(P3X63-AG8-U1) at a ratio of 10:1 using polyethylene glycol PEG 4000 (Sigma, St. Louis, MO, USA) as the fusogenic agent. The hybridomas were selected in HAT medium (IMDM supplemented with hypoxanthine, aminopterin and thymidine) containing 10\% FBS. Primary screening of hybridoma supernatants collected from each hybridoma was performed against the unfixed immunogen ROS17/2.8 CL\#7 using immunocytochemical staining as described previously [22]. Positive hybridomas were cloned two times using the standard limiting dilution procedure in the presence of $20 \mathrm{ng} / \mathrm{ml}$ of human recombinant IL-6 (Cell Signaling Technology ${ }^{\circ}$, Danvers, MA, USA).

\section{Hybridoma screening and preparation of MAbs}

Reactivity to cultured cells ROS17/2.8 CL\#7 cells were seeded and cultured in 96 multi-well plates (Falcon) $(150 \mu \mathrm{l} /$ well, $7.8 \times 10^{3}$ cells/well) and cultures reached $70-80 \%$ confluency at day 4 were utilized for immunocytochemical staining. Bone marrow-derived mesenchymal stem cells (BM-MSCs) obtained from the femur and tibia of 4 week female Lewis rats [23] were seeded into 35-mm dishes $(1 \times$ $10^{6}$ cells/dish) using $\alpha$-MEM containing $15 \%$ FBS. MSCs were replated in wells of 96 multi-well plates $\left(7.8 \times 10^{3}\right.$ cells/well), and were immunocytochemically stained with hybridoma supernatants at day 3 culture as described in this section "Immunocytochemical staining of unfixed culture cells". Bone marrow cells obtained from male SD rats were cultured as described previously [22]. Bone marrow cells were cultured in $\alpha$-MEM involving nucleosides (Life Technologies, Grand Island, NY, USA) containing $15 \% \mathrm{FBS}$ at a concentration of $1.5 \times 10^{6}$ cells $/ \mathrm{ml}\left(0.2 \times 10^{6}\right.$ cells/well) using 96 multi-well plates in the presence of $1 \times 10^{-8}$ M 1 $\alpha, 25$-dihydroxy-vitamin D3 (Enzo Life Science, Farmingdale, NY, USA), $10 \%$ of the heat treated, concentrated ROS17/2.8 cell-conditioned medium (htROSCM) [22] and $50 \mathrm{ng} / \mathrm{ml}$ RANKL. For bioassay experiments to evaluate the effect of antigen cross-linking, $10 \mu \mathrm{l}$ of each hybridoma supernatant was added to the above cultures for osteoclastogenesis. After 4 days of culture, cells were stained for tartarate resistant acid phosphatase using an acid phosphatase kit (Sigma, St. Louis, MO, USA). Cells having more than three nuclei were counted as osteoclastlike multinucleated cells. For assessment of A7 MAb immunostaining and bioactivity, cells were cultured under the same conditions as before except for using $\alpha$-MEM without nucleosides and $20 \mathrm{ng} / \mathrm{ml}$ RANKL for immunostaining and in the presence of different concentrations of A7 MAb or mouse IgG1, Kappa monoclonal isotype control (Abcam, Cambridge, UK) for bioactivity. Antibody purification A7 MAb Ascitis was prepared from BALB/cCr mice as described previously [22]. A7 MAb was purified from the ascitis using protein A affinity chromatography 
using Proteus A antibody purification kit (AbDSerotec ${ }^{\circledR}, \mathrm{A}$ Bio-Rad company, Oxford, UK) according to the manufacturer's protocol. The purified antibody was desalted and concentrated using Proteus $10 \mathrm{KDa}$ concentrator MWCO (polyethersulphone) ultrafiltration spinners. Primary osteoblast cultures Primary osteoblasts were prepared from calvariae of neonatal SD rats (2-days old) using a standard protocol [23]. In brief, collected calvariae, which were pooled in ice-chilled PBS and cut into pieces, were exposed to five sequential enzymatic digestions by use of $0.1 \%$ Collagenase type I and $0.2 \%$ Dispase II (Wako Pure Chemicals) as described previously [24].

\section{Cell proliferation assay and calcification of primary osteoblasts}

Cell proliferation assay For the cell proliferation assay (MTT assay), cells were seeded in 96 multi-well culture plates $\left(3.2 \times 10^{3}\right.$ cells $/ 100 \mu \mathrm{l} /$ well $)$ in $\alpha$-MEM containing $10 \%$ FBS and different concentrations of A7 MAb or control IgG. After cells reached confluency, cell proliferation assay was performed using cell counting kit-8 (CCK-8, Dojindo Molecular Technologies, Inc., USA) and absorbance was measured at $480 \mathrm{~nm}$ by use of Multiskan ${ }^{\mathrm{TM}} \mathrm{GO}$ microplate spectrophotometer (Thermo Fisher Scientific, MA, USA). Calcification of primary osteoblasts For differentiation and calcification of primary osteoblasts, cells were cultured in 96 multi-well culture plate $\left(1 \times 10^{4}\right.$ cells/ $\left.\mathrm{cm}^{2}\right)\left(3.2 \times 10^{3}\right.$ cells $/ 100 \mu \mathrm{l} /$ well $)$. On day 1 of culture, medium was replaced with osteogenic medium $(\alpha-\mathrm{MEM}$ containing $10 \% \mathrm{FBS}, 50 \mu \mathrm{g} / \mathrm{ml}$ ascorbic acid and $5 \mathrm{mM} \beta$ glycerophosphate). Osteogenic medium was replaced every 3 days and calcified deposits were stained with alizarin red at day 21 of culture, imaged by Keyence Biozero (BZ-8100) microscope.

\section{Immunohistochemical staining of rat tissue array}

Immunohistochemical screening was performed using rat tissue array, in which 60 different paraffinized tissue sections obtained from 4 rats were arrayed (MaxArray Rat Tissue Microarray slides, Zymed ${ }^{\circledR}$ Laboratories Inc., CA, USA). Deparaffinization and rehydration of the array was performed according to the manufacturer's instructions. The tissue array was then blocked with $5 \%$ chick albumin in PBS for $4 \mathrm{~h}$ at room temperature in a moisture chamber, and further blocking was performed using $10 \%$ goat serum in PBS overnight at $4{ }^{\circ} \mathrm{C}$. The array was then incubated with $\mathrm{A} 7 \mathrm{MAb}$ or PBS for $4 \mathrm{~h}$ at room temperature in moisture chamber. After rinsing with $\mathrm{PBS}$, the tissue array was further stained using ABC-AP kit (Vector Laboratories, Burlingame, CA, USA). Briefly, tissue array was incubated with biotinylated anti-mouse $\operatorname{IgG}(\mathrm{H}+\mathrm{L})$ followed by treating with ABC-AP complex. Color development was performed using Vector Red Color Development solutions in the presence of $1 \mathrm{mM}$ of levamisole to block endogenous alkaline phosphatase. The array was mounted in $90 \%$ glycerol. Microscopic observation was performed under Olympus AX70 microscope (Olympus, Tokyo, Japan). Immunofluorescence staining of unfixed cultured cells and observation by confocal laser scanning microscopy ROS17/2.8 CL\#7 and whole rat bone marrow cells were cultured at a density of $1.8 \times 10^{4}$ cells $/ \mathrm{ml}$ and $0.5 \times 10^{6}$ cells $/ \mathrm{ml}$ in a $35-\mathrm{mm}$ glass-bottom dishes (MatTek Co., Ashland, MA, USA) in $2 \mathrm{ml} \mathrm{DMEM,} \mathrm{10 \%}$ FBS and $\alpha$-MEM, $15 \%$ FBS respectively. After removal of the culture medium, cells were reacted with $\mathrm{A} 7 \mathrm{MAb}$, control $\mathrm{IgG}$ or PBS at $4{ }^{\circ} \mathrm{C}$ for $30 \mathrm{~min}$. The cells were then rinsed with serum-free medium and fixed in $4 \%$ paraformaldehyde in PBS ( $\mathrm{pH} 7.4)$ at room temperature for $20 \mathrm{~min}$. After rinsing and blocking with $3 \%$ goat serum in PBS for $30 \mathrm{~min}$ cells were reacted with goat anti-mouse IgG conjugated with Alexa Fluor 488 (Biolegend, San Diego, CA, USA) at room temperature for $30 \mathrm{~min}$ in the dark. Cells were then treated with $4^{\prime}, 6-$ diamidino-2-phenylindole (DAPI) (Sigma/Merk FGsA, Darmstadt, Germany) to stain nuclei for $5 \mathrm{~min}$ in dark. After rinsing in PBS, cells were observed by confocal laser scanning microscopy Nikon C2si (Nikon Instech, Tokyo, Japan). Differential interference contrast (DIC) images were also taken in addition to the immunofluorescence images. Two and three dimensional images of the cells were obtained and analyzed by the use of NIS-Elements AR 4.000.06 software (Nikon Instech, Tokyo, Japan).

\section{Flow cytometry and confocal laser analysis}

Confluent ROS17/2.8 cell clone \#7 was rinsed in PBS two times and trypsinized with $0.05 \%$ Trypsin, $0.02 \%$ EDTA at $37^{\circ} \mathrm{C}$ for $3 \mathrm{~min}$. Cells were passed through $70 \mu \mathrm{m}$ cell strainer and centrifuged at 2000rpm for $5 \mathrm{~min}$. After washing the cells in ice cold PBS and centrifuged, cell pellets were suspended in $100 \mu$ l of flow cytometry (FCM) buffer. Primary antibody (Hybridoma supernatant of isotype control IgG (mouse IgG1) or A7 MAb) was added followed by incubation at $4{ }^{\circ} \mathrm{C}$ for $45 \mathrm{~min}$. After addition of $1 \mathrm{ml}$ FCM buffer, cells were centrifuged at $1400 \mathrm{rpm}$ at $4{ }^{\circ} \mathrm{C}$ for $6 \mathrm{~min}$. After washing the cells in $1 \mathrm{ml}$ FCM buffer, centrifuged cells were resuspended in $100 \mu \mathrm{l}$ of FCM. Antimouse IgG conjugate with Alexa fluor 488 (Biolegend, PA, USA) diluted in 300 times was added and incubated in $4{ }^{\circ} \mathrm{C}$ for $30 \mathrm{~min}$. After washing the cells in FCM two times at $4^{\circ}$ $\mathrm{C}$, cells were suspended in FCM and applied for FACS analysis by use of FACSverse (Bickton Dickinson). 
Immunofluorescence confocal laser analysis of the frozen sections Four-week old female Lewis (LEW/SsN) rats were anaesthetized with isoflurane inhalation followed by i.p. injection of pentobarbital ( $1 \mathrm{mg} / \mathrm{Kg}$ body weight). The rats were aseptically dissected and fixed by perfusion of $4 \%$ paraformaldehyde (PFA) in PBS from the left ventricle. Excised bone tissue was decalcified in $10 \%$ EDTA at $4^{\circ} \mathrm{C}$ for 3 weeks. Decalcified bone tissue was embedded in OCT compound, frozen in dry-ice containing isopentane and stocked in $-80^{\circ} \mathrm{C}$ until use. Frozen sections were prepared using cryostat HM560MV (Zeiss, Oberkochen, Germany) and stocked in $-30^{\circ} \mathrm{C}$ until use, then processed for immunofluorescent staining. Sections were completely dried up at room temperature and immersed in PBS to remove the OCT compounds followed by washing in 10 $\mathrm{mM}$ glycine in PBS to quench unreacted aldehyde groups. Specimens were blocked in 5\% chicken albumin in PBS for $6 \mathrm{~h}$ at room temperature followed by overnight blocking in $10 \%$ goat serum in PBS in a moisture chamber at $4{ }^{\circ} \mathrm{C}$. Sections were incubated with A7 MAb or control IgG or $\mathrm{PBS}$ at room temperature for $2 \mathrm{~h}$ in a moisture chamber. After washing with PBS, sections were incubated with goat anti-mouse IgG polyclonal antibody conjugated with Alexa Fluor 488 (Biolegend, San Diego, CA, USA) diluted 300 times in $1 \% \mathrm{BSA}$ in PBS for $30 \mathrm{~min}$ in dark. Cells were then treated with DAPI for $5 \mathrm{~min}$ in dark. After rinsing in PBS, sections were mounted in Aqua-Poly/Mount (Polysciences Inc., Washington, PA, USA) for microscopic observation using confocal laser scanning microscopy (Nikon Instech, Tokyo, Japan).

\section{Western blotting}

Western blotting was carried out using a semi-dry procedure. Cultured cells were lysed in Radio-immunoprecipitation Assay (RIPA) buffer (150 mM NaCl, 1\% v/v NP-40, 0.5\% DOC, $50 \mathrm{mM}$ Tris- $\mathrm{HCl}$ (pH 8.0), 0.1\%SDS) containing protease inhibitor mixture P1860 (Sigma, St. Louis, MO, USA) on ice for $30 \mathrm{~min}$ and subjected to $10 \%$ SDS-PAGE followed by transfer to PVDF membrane (GH Healthcare, Chicago, IL, USA). Protein concentration of cell lysates was determined by Bio-Rad protein assay reagent (Bio-Rad, Hercules, CA, USA). After blocking with 5\% non-fat dry milk for $1 \mathrm{~h}$, membranes were reacted overnight with primary antibodies A7 MAb or control IgG diluted with 5\% non-fat dry milk at $4{ }^{\circ} \mathrm{C}$. After washing with TBS-tween three times, the membrane was treated with HRP-conjugated anti-mouse IgG antibody (Cell Signaling Technology, USA) as the secondary antibody for $1 \mathrm{~h}$. After washing with TBS-tween $(0.1 \%)$ three times, immunoreactive bands were detected by use of luminogenic reagent ECL ${ }^{\mathrm{TM}}$ Prime Western Blotting Detection Reagent (GE Healthcare) and imaged by use of ImageQuant Las 4000 (GH Healthcare).

\section{Biotinylation of cell surface proteins and immunoprecipitation}

Cell surface proteins of ROS17/2.8 CL\#7 cells were biotinylated using ECL ${ }^{\mathrm{TM}}$ Protein biotinylation kit (GH Healthcare) according to the manufacturer's protocol. Cell surface proteins of confluent culture of ROS17/2.8 CL\#7 cells were biotinylated at $4{ }^{\circ} \mathrm{C}$. After rinsing in ice-chilled PBS, cell lysates were prepared by use of the RIPA buffer containing protease inhibitor cocktail the same lysis buffer as utilized in Western blotting. Immunoprecipitation was performed using Immunoprecipitation kit-Dynabeads ${ }^{\circledR}$ Protein G (Invitrogen, Oslo, Norway) according to the manufacturer's protocol. Briefly, Dynabeads were bound with $10 \mu \mathrm{g}$ of A7 MAb or isotype control $\mathrm{IgG}$ for $10 \mathrm{~min}$ at room temperature. Dynabeads-antibody complex was incubated with cell lysates prepared from biotinylated ROS17/2.8 CL\#7 cells for $10 \mathrm{~min}$ at room temperature with mild mixing. Dynabeads-antibodybiotinylated antigen complex was rinsed and eluted according to the manufacturer's protocol. Eluted proteins were mixed with a pre-mixed SDS-sample buffer $5 \mathrm{x}$ and 2mercaptoethanol followed by heating for $10 \mathrm{~min}$ at $70^{\circ} \mathrm{C}$. The samples were subjected to SDS-PAGE using $10 \%$ gel followed by transfer to PVDF membrane. After blocking in $5 \%$ non-fat dry milk for $1 \mathrm{~h}$, the membrane was incubated with streptavidin-HRP conjugate (GH Healthcare) for $1 \mathrm{~h}$ at room temperature. After washing the membrane in TBStween, biotinylated protein bands were detected by use of luminogenic reagent ECL ${ }^{\text {TM }}$ Prime Western Blotting Detection Reagent (GE Healthcare, UK) and imaged by ImageQuant Las 4000 (GE Healthcare). Subclass determination The isotype of the A7 MAb was determined by use of mouse monoclonal antibody isotyping kit (IsoStrip, Roche Applied Science, Indianapolis, USA) according to the manufacturer's instructions.

\section{Statistical analysis}

All data are represented as the mean \pm SEM from at least three independent experiments. Statistical analysis was performed by Student's $t$-test and ANOVA. $P<0.05$ was considered as statistically significant.

\section{Results}

\section{Generation of the osteoblast-reactive monoclonal antibodies (MAbs) affecting osteoclastogenesis}

In the present study, we generated a panel of novel MAbs raised against cell-surface membrane molecules expressed by a clone of the rat osteoblastic cell line ROS17/2.8 cells. Unfixed ROS17/2.8 CL\#7 cells were utilized for mice 
immunization and a series of antibodies was established. Supplemental Table 1 shows the results of the primary screening of MAbs. Although we utilized osteoblastic cell line ROS17/2.8 CL\#7 as the immunogen, various patterns of antibody specificity was observed. A7 and A33 showed intense reactivity to ROS17/2.8 CL\#7 with mild (A7) or no (A33) reactivity to MSCs and bone marrow stromal cells. A67, A106 and A111 were preferentially reactive to MSCs with mild reactivity to ROS17/2.8 CL\#7 and bone marrow stromal cells. Other antibodies showed a staining pattern of nonspecific binding, in which antibodies were reactive to both osteoblast-lineage and osteoclast-lineage cells. Nonspecific antibodies tended to show regulatory activity on osteoclastogenesis. As A33 is highly specific to ROS17/2.8 CL\#7 cells but not reactive to bone marrow stromal cells or MSCs, it might be specific to osteosarcoma. In contrast, A7 was strongly reactive to ROS17/2.8 CL\#7 and mildly reactive to bone marrow-derived MSCs and osteoblastic bone marrow stromal cells formed in cultures for forming osteoclasts [23] with no reactivity to osteoclasts. Therefore, A7 antibody appears to be specific to cells of osteoblastlineage. Our primary purpose of the current study was to investigate unique regulatory molecules specifically expressed on cell surface of osteoblasts. Although no significant bioactivity on osteoclastogenesis was detected in A7 antibody in the first screening of hybridoma supernatants, we selected A7 antibody to search for osteoblastlineage specific cell surface molecules bearing some regulatory function in bone remodeling. We selected A7 hybridoma for an extensive cloning by the standard sequential limiting dilution method and obtained pure hybridoma producing monoclonal antibody A7 MAb highly specific to osteoblasts. We examined immunoglobulin subtype of the antibody and determined its subtype as mouse IgG1 with kappa light chain. This A7 MAb was highly specific to cells of osteoblast-lineage.

\section{Expression of A7 antigen on cell surface of osteoblastic cells}

Initially, we confirmed the reactivity of A7 MAb to unfixed osteoblastic cells ROS17/2.8 CL\#7. Immunofluorescent detection of A7 antigen was performed at day 4 of culture. A7 antigen seemed to show a typical cell surface expression pattern (Fig. 1). Although antigen expression in areas of lower cell density showed weaker immunofluorescence signal (Fig. 1a), the level of immunofluorescence signal showing A7 antigen expression was quite high in the areas of higher cell density (Fig. 1b). Isotype control IgG showed no immunofluorescence signal (Fig. 1c). These data show that expression of A7 antigen is dependent on cell density. Similar high expression of A7 antigen was observed in the original ROS17/2.8 cells before cloning and in other ROS17/2.8 cell clones (data not shown). We examined the precise pattern of A7 antigen expression of this ROS17/2.8 cell clone by use of confocal laser microscope. Figure 2a shows the distribution of A7 antigen at one specified section parallel to the bottom of the culture dish. Most of the cells showed the typical membrane distribution pattern of A7 antigen, in which immunofluorescence signal is localized to the cell membrane. In addition to the typical membrane distribution pattern, dot-like immunofluorescence signal was also detected in some cells. As we treated unfixed cells with A7 MAb to detect A7 antigen, the dot-like signal was likely related to internalized A7 antigen. To confirm this possibility, three-dimensional analysis was performed. Figure $2 b$, c, d show threedimensional images of the A7 antigen distribution from different directions. In addition to the cell surface localization of A7 antigen, we observed dot-like signals of A7 antigen at the apical cell surface of some cells, suggesting the presence of numerous internalized A7 antigen detected in dot-like structures possibly related to endosomes. Supplemental Fig. 1 shows confocal laser microscopic sections taken at various cell heights, starting from the basal cell surface attaching the culture dish till the apical surface away from the culture dish as indicated as level 1-16 shown in supplemental Fig. 1a. Supplemental Fig. 1b shows that typical membrane localization pattern of A7 antigen was detected in various heights of the cells. Near the basal side (level 3), granular pattern of A7 antigen was detected in addition to the marginal localization pattern of this antigen (supplemental Figure 1b3). At higher levels, typical membrane localization pattern of A7 antigen was observed (level 4-15, supplemental Figure 1b4-15) while some other cells showed dot-like signals at their apical cell surfaces away from the culture dish (level 7-9, supplemental Figs. 1b7-9). These observations strongly suggest that the A7 antigen is the cell surface molecule. Supplemental Fig. 2 shows the expression of osteoblast marker Alkaline Phosphatase (ALP) in ROS17/2.8 cell clone\#7, suggesting A7 antigen expressing cells are really osteoblasts. Among antibody panel of Supplemental Table 1, A47 antibody does not react to osteoblasts. Supplemental Fig. 3 shows the reactivity of A47 antibody to ROS17/2.8 cells. These data confirm the high specificity of A7 MAb against osteoblastic cell line ROS17/2.8 cells.

We also investigated the expression of A7 antigen on primary osteoblasts obtained from calvaria of newborn rats. As shown in Supplemental Fig. 4, primary osteoblasts expressed high level of A7 antigen in a similar membrane pattern as ROS17/2.8 cells.

\section{Expression of A7 antigen on cell surface of osteoblastic cells analyzed by FACS}

In order to obtain more definitive data concerning the expression of A7 antigen on cell surface of osteoblasts, we 
Fig. 1 Expression of A7 antigen on cell surface of osteoblastic cell line ROS17/2.8. CL\#7 cells were cultured in $35 \mathrm{~mm}$ glassbottom dishes $\left(1.8 \times 10^{4}\right.$ cells/ $\mathrm{ml}, 3.6 \times 10^{4}$ cells/dish) for 4 days. Unfixed cells were stained with $\mathrm{A} 7 \mathrm{MAb}(\mathbf{a}, \mathbf{b})$ or isotype $\operatorname{IgG}(\mathbf{c})$ as the the primary antibody as described in Materials and Methods. After rinsing, fixing and blocking, cells were treated with antimouse IgG conjugated with Alexa Fluor 488 (green). Nuclei were stained with DAPI (blue). a A7 antigen expression in areas of lower cell density detected by A7 MAb. b A7 antigen expression in areas of higher cell density. c Reactivity of isotype control IgG on ROS17/2.8 CL\#7 cells. Bars, $50 \mu \mathrm{m}$
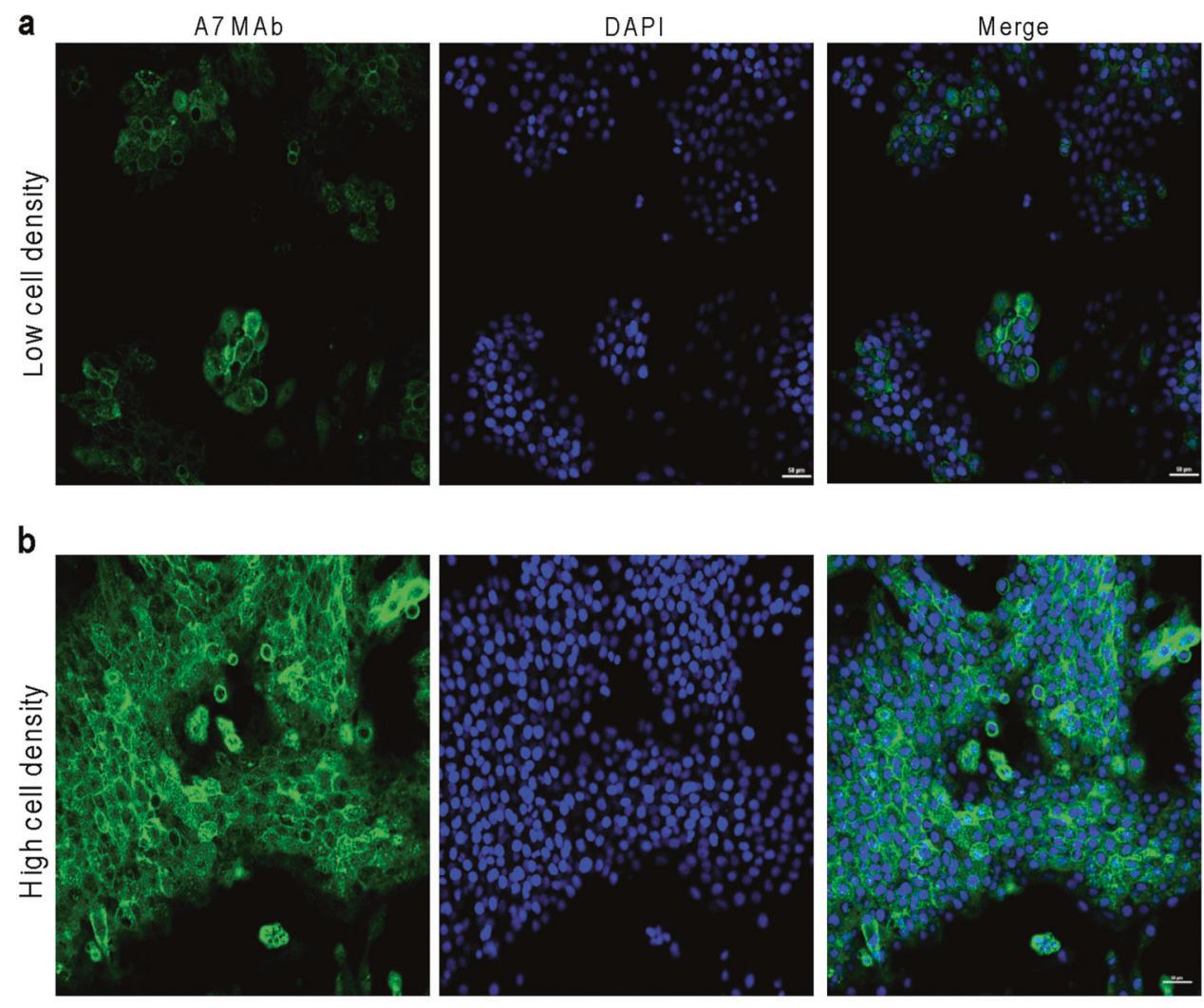

c Isotype control lgG
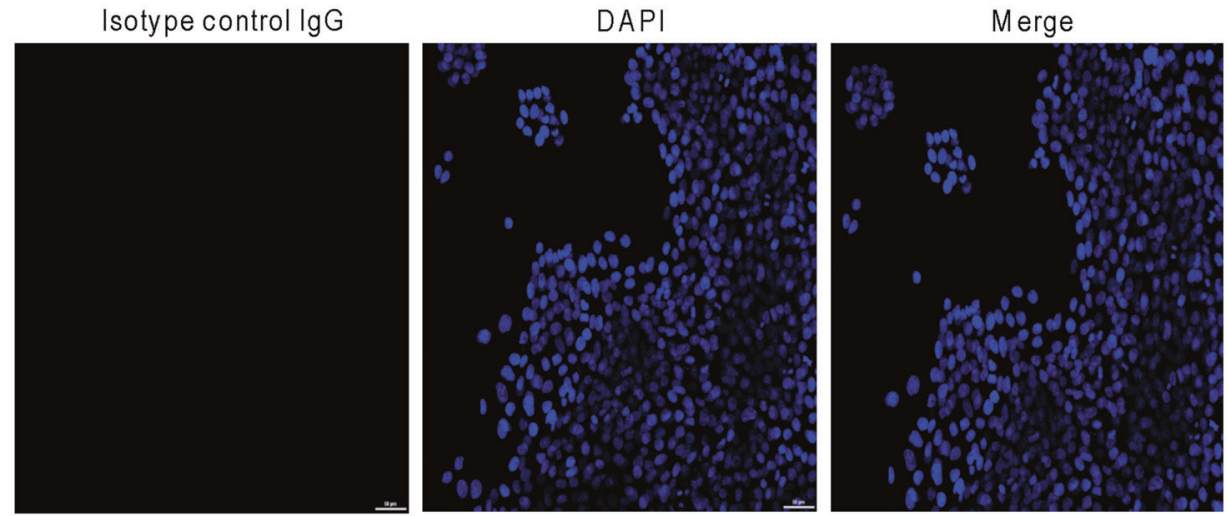

performed flow cytometry analysis. As shown in Fig. 3, A7 antigen-positive cell population was clearly detected. We conclude that A7 antigen is really expressed on cell surface of osteoblasts.

\section{Expression of A7 antigen in bone marrow stromal cells in cultures for osteoclastogenesis and in bone tissues}

In whole bone marrow cultures stimulated for osteoclastogenesis, cells were immunofluorescently stained with A7 $\mathrm{MAb}$ or control IgG. Bone marrow stromal cells demonstrated strong reactivity to A7 MAb (Fig. 4). However, the reactivity was much stronger at the sites of cellular aggregations (Figs. 4a, b). Observing the stromal cells at high magnification view revealed intense cell surface staining of mononuclear, spindle-shaped or polygonal cells and weaker staining of their cellular processes (Fig. 4b). As no reactivity was detected with multinuclear cells, we assumed that A7 MAb specifically reacted to the cells in the mesenchymal lineage and does not react to hematopoietic multinuclear osteoclasts or their mononuclear precursors which are hematopoietic in origin. In order to confirm the immunofluorescence data, we performed immunocytochemical staining of whole bone marrow cultures for osteoclastogensis.

Monoclonal antibody Kat1 (Kat1 MAb) recognizes the antigen specifically expressed on cell surface of osteoclasts 
Fig. 2 Expression pattern of A7 antigen in osteoblastic cell line observed by two and threedimensional confocal microscope imaging. ROS17/2.8 CL\#7 cells were cultured and stained with A7 MAb as described in the legends of Fig. 1. a Expression and distribution pattern of $\mathrm{A} 7$ antigen detected by $\mathrm{A} 7 \mathrm{MAb}$ in a horizontal section of ROS17/ 2.8 CL\#7 cells parallel to the bottom of the culture dish. Bars, $10 \mu \mathrm{m}$. b-d Three dimensional images of A7 antigen expression detected by A7 MAb in ROS17/ 2.8 CL\#7 cells from different views (A7 MAb/DAPI merge). Three-dimensional images were reconstructed from confocal images of each height shown in supplemental Fig. 1. b Top view showing A7 antigen expression on apical and lateral cell surfaces. c Bottom view showing the basal cell surface attaching the culture dish. $\mathbf{d}$ Top and side view. Bars, $10 \mu \mathrm{m}$
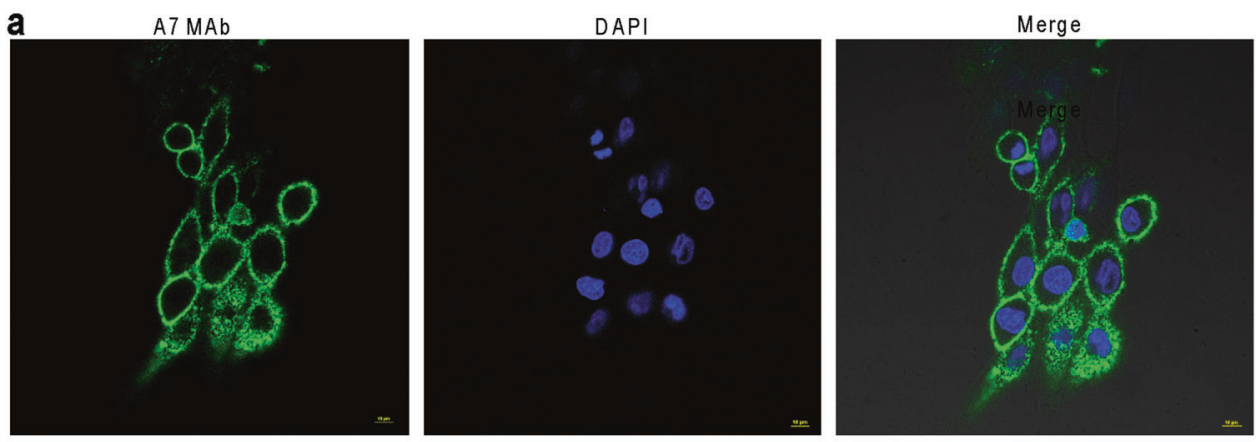

b

C
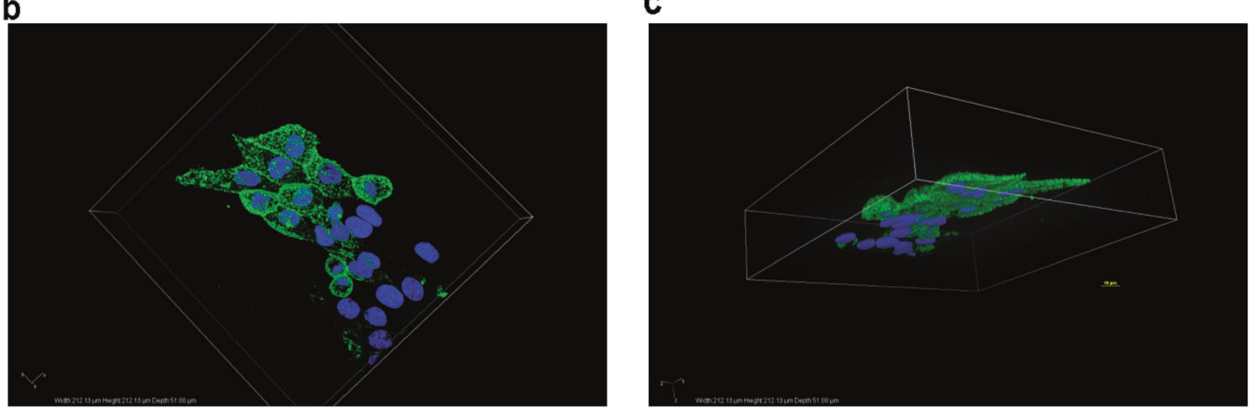

d

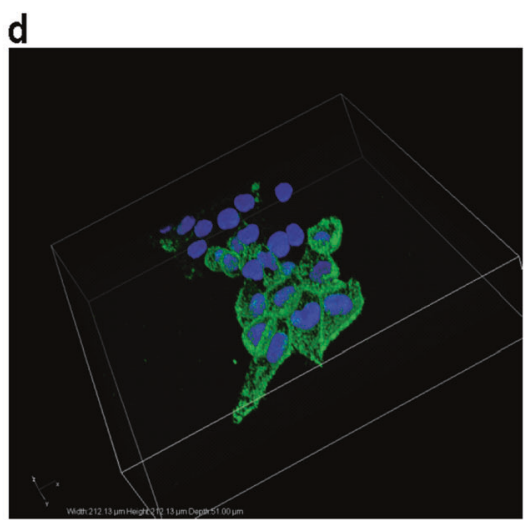

[22, 25-27]. For the purpose of comparison, we utilized A7 $\mathrm{MAb}$ and the osteoclast-specific Kat1 MAb for immunocytochemical staining (Fig. 5). A7 MAb intensely stained bone marrow stromal cells in culture for osteoclastogenesis, however it did not stain osteoclast-like MNCs (Fig. 5a; arrowheads). In contrast, Kat1 MAb showed a specific immunoreactivity to multinucleated osteoclasts (Fig. 5b; arrowheads) without any reactivity to bone marrow stromal cells present in cultures of osteoclastogenesis (Fig. 5b). These data clearly show that A7 MAb recognizes the antigen specifically expressed on cells in the mesenchymal lineage but never react to cells in the hematopoietic lineage involving osteoclasts, confirming the highest lineage specificity of A7 MAb. Expression of A7 antigen in osteoblasts in vivo Next we examined the specificity of A7 antigen expression in bone tissue by use of sections of femur and tibia. Figure 5 shows immunoreactivity of A7 MAb in the cryosection of proximal tibia. A7 MAb demonstrated specific immunoreactivity with cuboidal cells covering the bone trabeculae observed in the metaphysis. Clear membrane pattern of A7 antigen expression was observed in the cuboidal cells. No immunoreactivity was observed in the growth plate chondrocytes as well as in bone marrow (Fig. 6a, Fig. 6b upper panels). Figure 6a (right panel) shows the similar field view of the serial section stained with hematoxylin and eosin (HE). A7-positive cuboidal cells observed on bone surface are likely to be typical osteoblasts. In the cortical bone of tibial diaphysis, A7 antigen was markedly expressed in surface periosteal cuboidal or polygonal-shaped cells. A7 antigen was also expressed in osteocytes (Fig. 6b, lower panels). To determine whether A7-positive cells observed on the bone surfaces were osteoblasts, serial sections of A7 MAb stained sections were stained for ALP activity. The expression pattern of $\mathrm{A} 7$ antigen was almost coincident with that of ALP, however some cells were positive for ALP without 

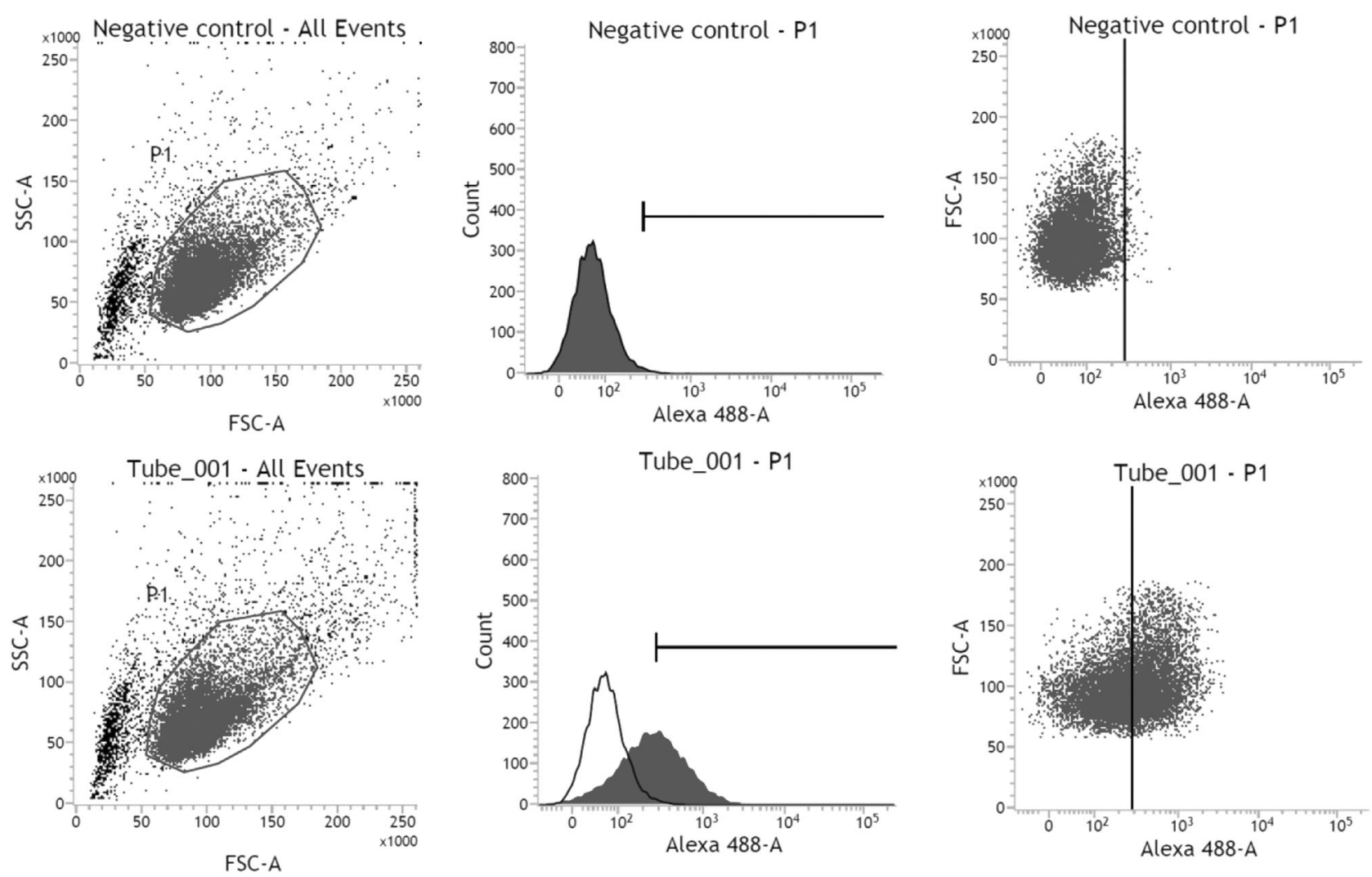

Fig. 3 Cell-surface expression of A7 antigen in osteoblastic cell line revealed by flow cytometry analysis. Confluent ROS17/2.8 CL\#7 cells were suspended by a short trypsinization and stained with A7 MAb or control IgG followed by staining with a second antibody, anti-mouse

IgG conjugated with Alexa Fluor 488, as described in Materials and Methods. The cells were analyzed by FACS. FSC forward scatter, SSC side scatter. Upper panels: Isotype control IgG, Lower panels: A7 $\mathrm{MAb}$

expressing A7 antigen (Fig. 6c). These results demonstrate that A7 antigen is specifically expressed on the plasma membrane of a subpopulation of cells of osteoblast lineage.

\section{Demonstration of the specificity of A7 antigen expression using tissue array}

In order to assess the specificity of A7 antigen expression, we stained tissue arrays. Intense immunoreactivity was observed specifically in bone tissue. Although moderate reactivity was observed in lung tissue, A7 MAb showed no reactivity to other tissues, including heart muscle, liver, spleen, kidney, central nervous system, muscle, tongue, skin, stomach, small bowel, ovary/testis, and urinary bladder. Figure 7 shows A7 MAb staining on paraffin sections of bone tissue. Intense immunoreactivity was observed in osteocytes present in shallow areas just close to bone surface. In contrast to the results of frozen sections, immunoreactivity to bone surface osteoblasts bearing cuboidal morphology was weak; however, intense immunoreactivity was observed in osteocytes and some bone surface osteoblasts (Fig. 7a, arrowheads in the right panel). Figure 7b shows the similar field view of the serial section of Fig. 7 a stained with HE. Osteocytes present just close to the bone surface showed intense membrane staining pattern. In contrast, osteocytes present away from the bone surface showed no immunoreactivity (Fig. 7c). These data strongly suggest that the antigen is preferentially expressed in newly formed osteocytes but not in old osteocytes embedded in old bone matrices. Clear immunoreactivity of A7 MAb to osteocytes was also observed in Fig. 6b (lower panel) and Fig. $6 c$ in which frozen sections were utilized, confirming the expression of A7 antigen in osteocytes.

\section{Lineage and species specificity of A7 antigen expression analyzed by immunoblotting}

Figure 8a shows the western blotting analysis of A7 antigen in cell lysates of ROS17/2.8 CL \#7. Although IgG isotype control antibody detected no protein band (lane 1), A7 MAb specifically reacted to a clear protein band of approximate molecular weight of $45 \mathrm{KDa}$ (lane 2) (Fig. 8a). Figure 8b shows western blotting of cell lysates prepared from various cell lines. A7 antigen was expressed in rat osteoblastic cell lines ROS17/2.8 CL \#7 and UMR106. A7 antigen was also expressed in rat myoblast cell line L8. A7 antigen was not 
Fig. 4 Expression of A7 antigen in bone marrow stromal cells Bone marrow cells of 4-weekold SD male rats were cultured in $35 \mathrm{~mm}$ glass-bottomed dishes $\left(1 \times 10^{6}\right.$ cells/dish $)$ for forming osteoclasts as described in Materials and Methods. Unfixed cells were stained with A7 MAb and detected by anti-mouse IgG conjugated with Alexa Fluor 488 (green) as described in legends of Fig. 1. Nuclei were stained with DAPI (blue). a A7 antigen was expressed on bone marrow stromal cells at areas of high cell density. Upper and lower panels show different fields. Left: A7 MAb, center: A7 MAb/DAPI merge, right: DIC. Bars, $50 \mu \mathrm{m}$. b High magnification of mononuclear bone marrow stromal cells showing cell-surface expression pattern of A7 antigen. Left: A7 MAb, center: A7 MAb/DAPI merge, right: DIC. Bars, $10 \mu \mathrm{m}$
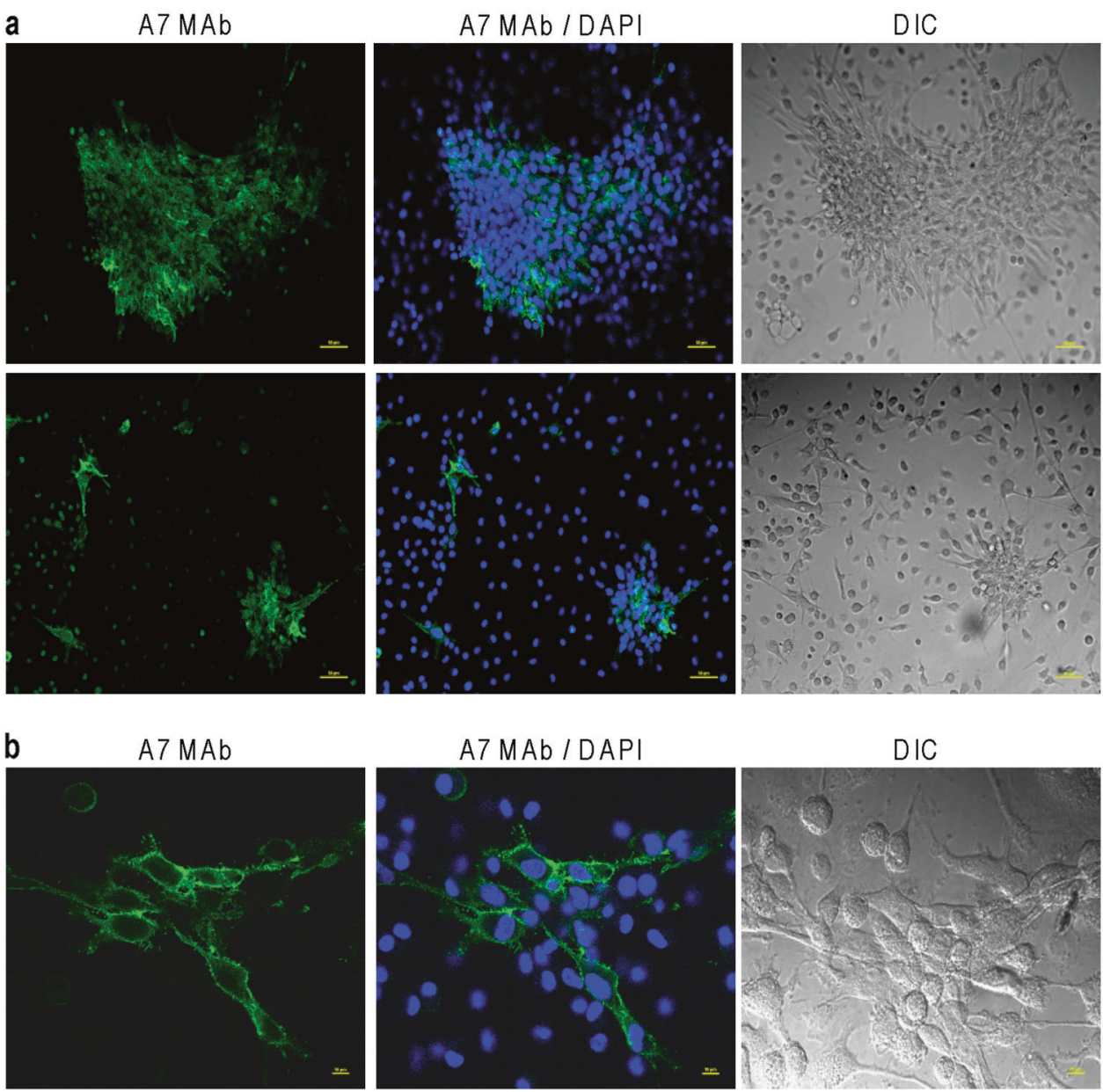

expressed in rat alveolar macrophage cell line NR8383. No protein band was detected in the mouse calvarial-derived osteoblastic cell line MC3T3-E1 or human osteoblastic cell lines Saos-2 and MG-63. No band was detected in murine osteoclast precursor cell line RAW-D cells treated with or without RANKL. These data suggest that A7 antigen is exclusively expressed in rat osteoblastic cell lines and a myoblast cell line.

Figure $8 \mathrm{e}$ shows the quantitative data concerning the time-course expression of the A7 antigen. Expression of A7 antigen was dramatically induced on day 3 of the culture when cells reached to be subconfluent, while protein level of $\beta$-tubulin did not change throughout culture period. These results are consistent with the immunostaning data shown in Fig. 1.

\section{Detection of cell surface A7 antigen expressed on osteoblasts by immunoprecipitation}

The membrane surface proteins of unfixed ROS17/2.8 CL\#7 cells were labeled with biotin. Figure 7c shows total cell surface biotinylated proteins extracted from biotinylated unfixed ROS17/2.8 CL\#7 cells. Numerous biotinylated proteins ranging from $300 \mathrm{KDa}$ to $10 \mathrm{KDa}$ were detected. Cell lysates containing the biotinylated cell-surface proteins were subjected to immunoprecipitation by use of control IgG or A7 MAb. As shown in Fig. 8d, immunoprecipitated protein with an approximate molecular weight of $45 \mathrm{KDa}$ was clearly detected by A7 MAb while no band was detected in lane of IgG isotype control. These data confirm that A7 antigen is a cell surface protein of approximate molecular weight $45 \mathrm{KDa}$.

\section{Cross-linking of A7 antigen stimulates osteoclast formation in vitro}

To investigate an involvement of A7 antigen specifically expressed on surface of osteoblasts in the regulation of osteoclastogenesis, we examined the cross-linking of A7 antigen by use of the anti-A7 antigen MAb, A7 MAb, on osteoclastogenesis in rat whole bone marrow cultures. A7 MAb significantly augmented osteoclast formation at a limited range of antibody concentrations (Fig. 9). When the osteoclast number was low, A7 MAb stimulated osteoclastogenesis at $10 \mu \mathrm{g} / \mathrm{ml}$ (Fig. 9a), while A7 MAb stimulated osteoclastogenesis at $0.5 \mu \mathrm{g} / \mathrm{ml}$ if number of 

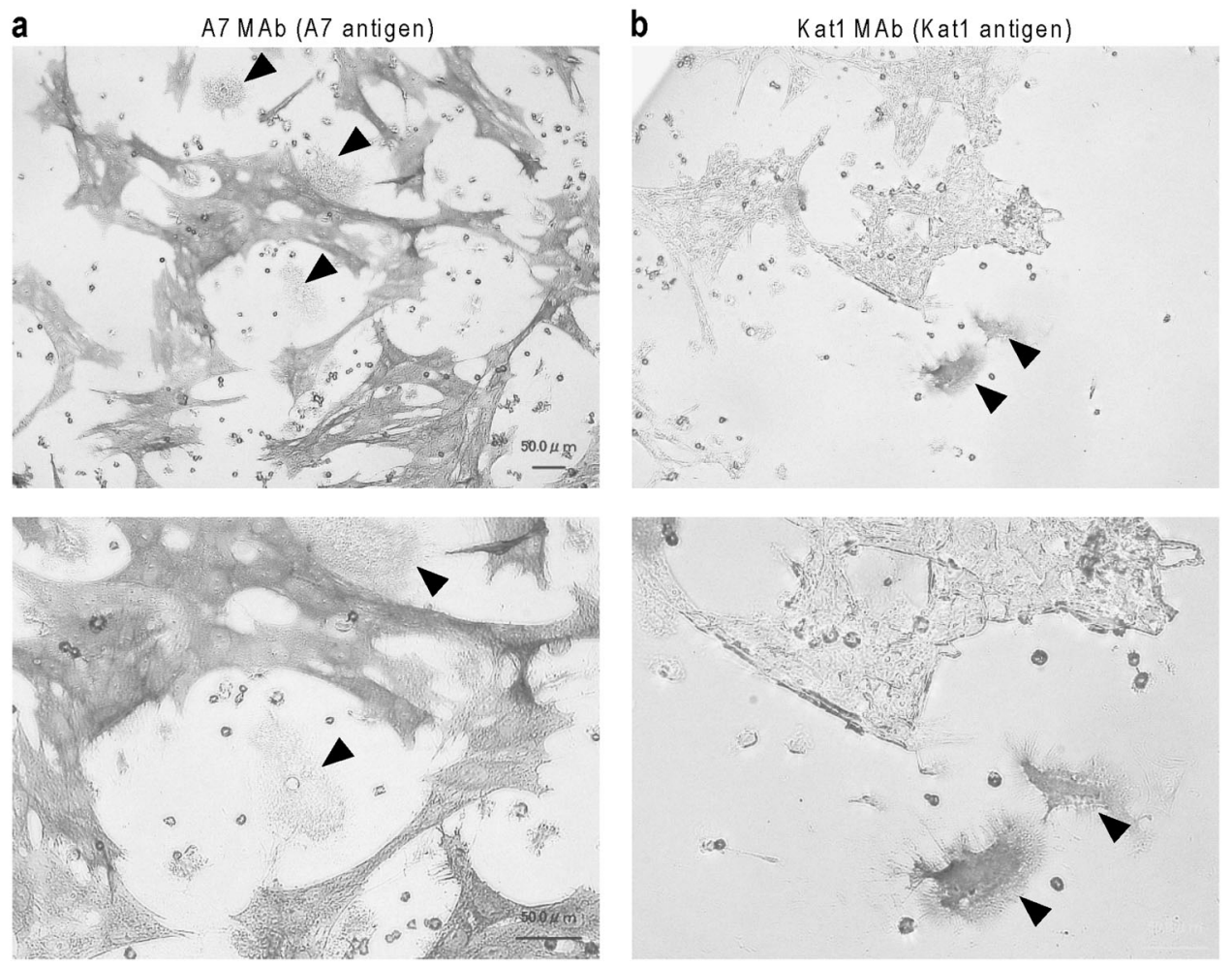

Fig. 5 Lineage specificity of A7 MAb in bone marrow cultures for osteoclastogenesis. Bone marrow cells of 4-week-old SD male rats were cultured for 4 days in 96 multi-well plates at a concentration of $\left(0.2 \times 10^{6}\right.$ cells/well $)$ as described in Materials and Methods. Unfixed cells were stained with $\mathrm{A} 7 \mathrm{MAb}$ or Kat-1 MAb as the primary antibody and further detected by ABC-AP kit (Vectastatin , Alkaline Phosphatase Standard, AK-5000) as described in Materials and Methods. Color development was performed using Vector Red

(Vector Red Alkaline Phosphatase substrate kit SK-5100) in the presence of $1 \mathrm{mM}$ levamisole. Lower panels are the higher magnification view of the upper panels. a Specific staining of bone marrow stromal cells with A7 MAb (red stained cells). Arrowheads: Osteoclast-like MNCs expressing no A7 antigen. b Specific expression of Kat1 antigen in osteoclasts. Arrowheads: Osteoclast-like MNCs specifically stained with Kat-1 MAb (red). Bars, $50 \mu \mathrm{m}$

osteoclasts was high (Fig. 9b). As the isotype control $\operatorname{IgG}$ showed no significant effects on osteoclastogenesis (Fig. 9c), binding of A7 MAb to cell surface A7 antigen results in a slight but significant augmentation in osteoclast formation.

\section{A possible regulatory effect of A7 MAb on calcification by osteoblasts}

In order to elucidate involvement of A7 antigen on osteoblast maturation and function, we examined the effect of A7 $\mathrm{MAb}$ on calcification mediated by calvarial-derived primary osteoblasts. As shown in Fig. 10a, A7 MAb markedly inhibited formation of calcified nodules, while the isotype control IgG did not affect deposition of calcified nodules. A7 MAb and control IgG did not affect proliferation and viability of primary osteoblasts as evaluated by the MTT assay as described in Materials and Methods (Fig. 10b). These results show a possible role for A7 antigen in the maturation of primary osteoblasts to form calcified matrices. Figure. 11 (upper panel) shows the summary of the

biological effect of anti-A7 antigen MAb on osteoclastogenesis and calcification mediated by osteoblasts. Crosslinking of cell-surface A7 antigen stimulated osteoclastogenesis and markedly suppressed calcification.

\section{Discussion}

The current study describes a unique cell-surface molecule specifically expressed on cells of osteoblast lineage involved in the precise regulation of bone remodeling. The anti-A7 antigen monoclonal antibody that we produced for this study binds to a cell surface antigen (A7 antigen) specifically expressed in cells of osteoblast lineage. We confirmed the cell-surface expression of A7 antigen on osteoblastic cell line by use of flow cytometry. We postulated that osteoblasts and bone lining cells have regulatory functions that determine the initiation sites for bone remodeling and the potential ability to accept signals for osteoblast formation and maturation. Therefore, we carried out an immunological survey to search for novel 
Fig. 6 Expression of A7 antigen in bone tissue. Longitudinal sections of tibia were prepared from 4-week-old female Lewis rats as described in Materials and Methods. Frozen tissue sections were prepared for staining. Sections were incubated with A7 MAb and detected with anti-mouse IgG conjugated with Alexa Fluor 488 (green) as described in Materials and Methods. a Metaphyseal region of proximal tibia. (Left panel) Staining with A7 MAb, (center panel) merged A7 MAb staining with DIC, (right panel) HE staining of the serial section. $C A$ cartilage, $T b$ trabecular bone, $B M$ bone marrow. b A higher magnification of Fig. 5a left and center panel. A7 antigen expression (left panels), merged A7 antigen expression and DIC (right panels). (Upper panels): Metaphyseal region of proximal tibia, (Lower panels): Cortical bone of the diaphyseal region of tibia. $B$ bone, $T b$ trabecular bone, $B M$ bone marrow, $C A$ cartilage. Arrowheads: typical cuboidal cells expressing A7 antigen in a typical membrane localization pattern. Arrows: osteocytes. Bars, $50 \mu \mathrm{m}$. c Metaphyseal region of proximal tibia showing A7 antigen signal in bone surface cells and osteocytes (left panel) and ALP staining of the similar field view of the serial section (right panel). Arrowheads: Double positive cells expressing A7 antigen and ALP. Arrows: Single positive cells expressing only ALP. $B$ bone, $B M$ bone marrow a
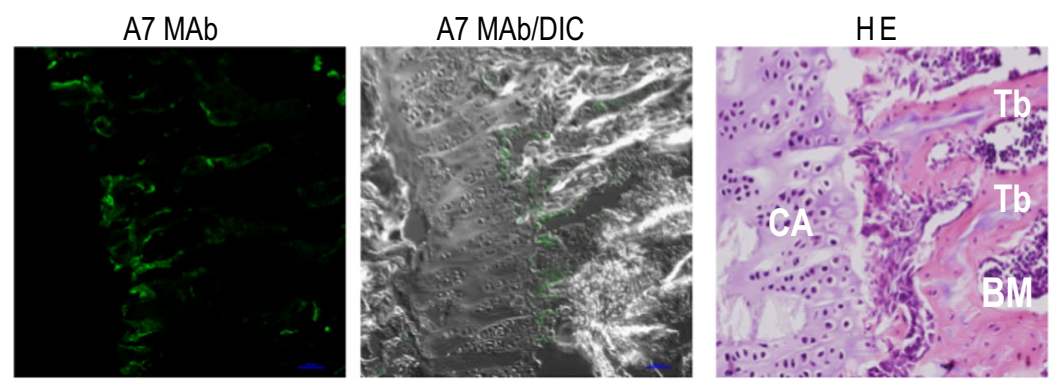

b
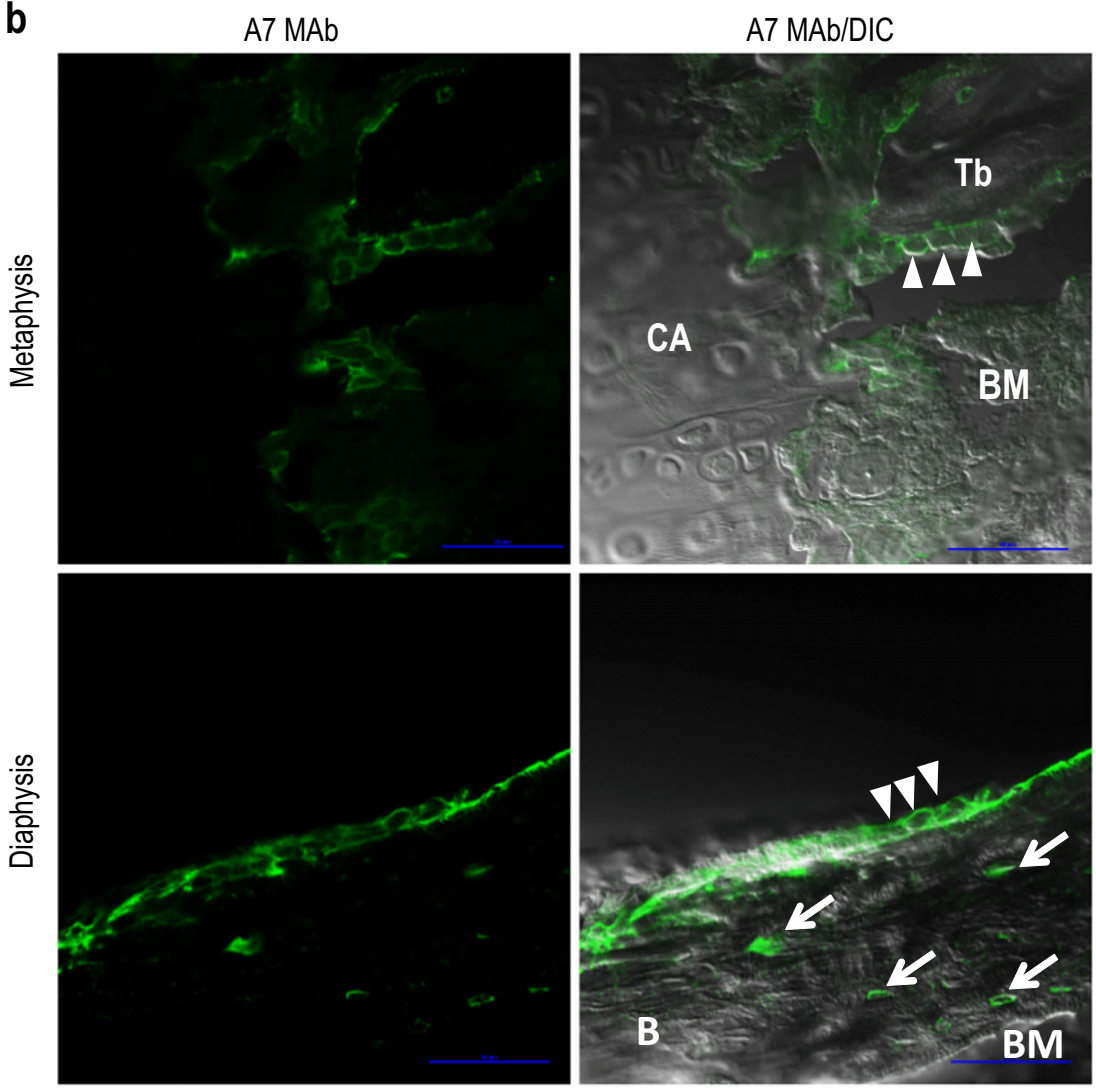

c

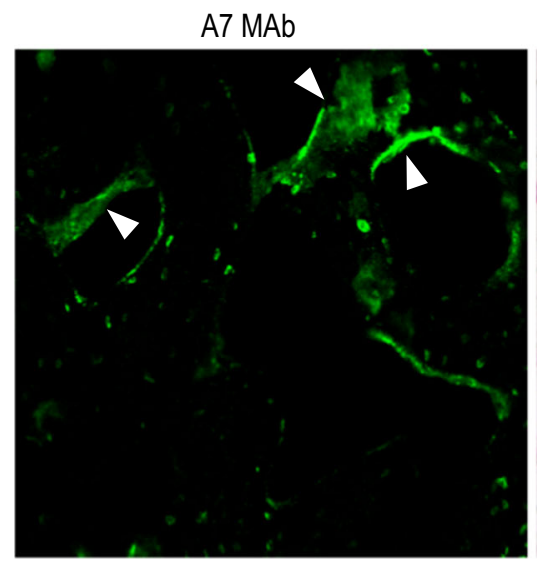

Alkaline Phosphatase

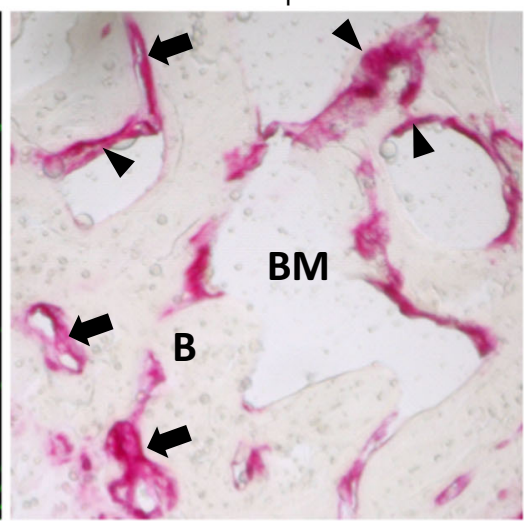

osteoblast-specific cell-surface molecules involved in the regulation of bone remodeling. We found the novel osteoblast-specific cell surface protein, A7 antigen, with the approximate molecular weight of $45 \mathrm{KDa}$. Crosslinking of cell surface A7 antigen by use of A7 MAb markedly suppressed calcification mediated by primary 
Fig. 7 Specific expression of A7 antigen in bone tissue: Tissue array study. Rat tissue arrays were deparaffinized and stained with A7 MAb as described in Materials and Methods. a Right panels are higher magnification views of the left panels. A7 antigen expression in osteocytes in bone tissue (red color). A7 antigen was specifically detected in osteocytes present just close to the bone surface and in the bone surface osteoblasts (arrow heads) in a membrane distribution pattern. b Similar field view of the serial section of (a) stained with HE. c Demonstration of the A7 antigen-positive osteocytes located just close to the bone surface and A7-negative osteocytes in deeper layers of bone. $B$ bone, $B M$ bone marrow. Bars, $50 \mu \mathrm{m}$ a
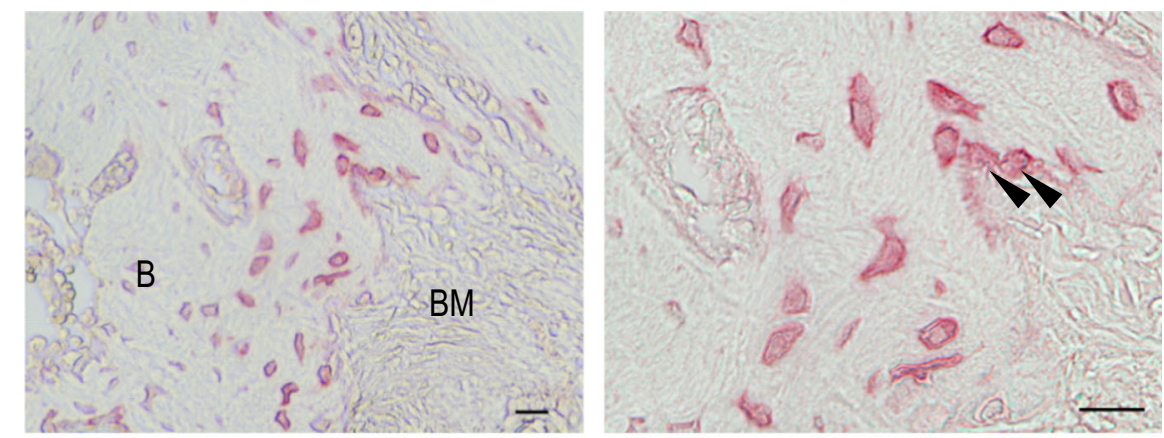

b

HE
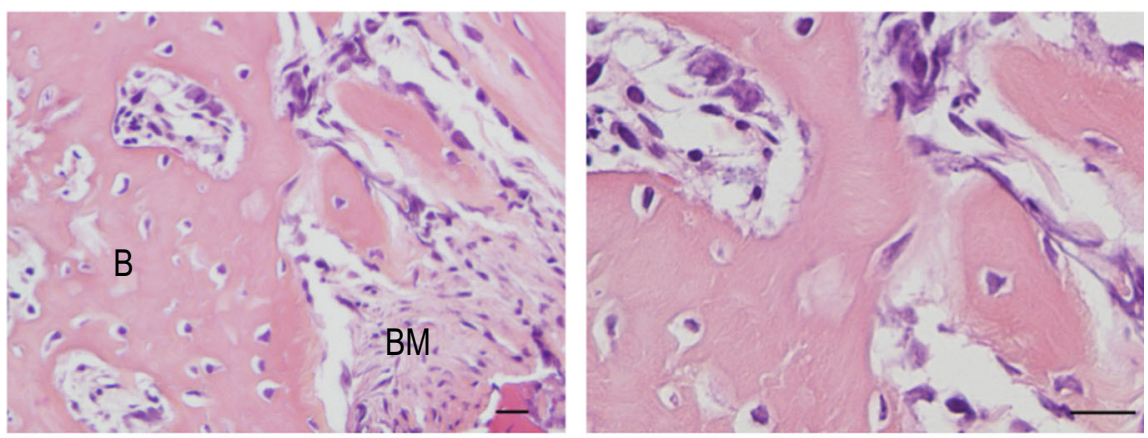

C
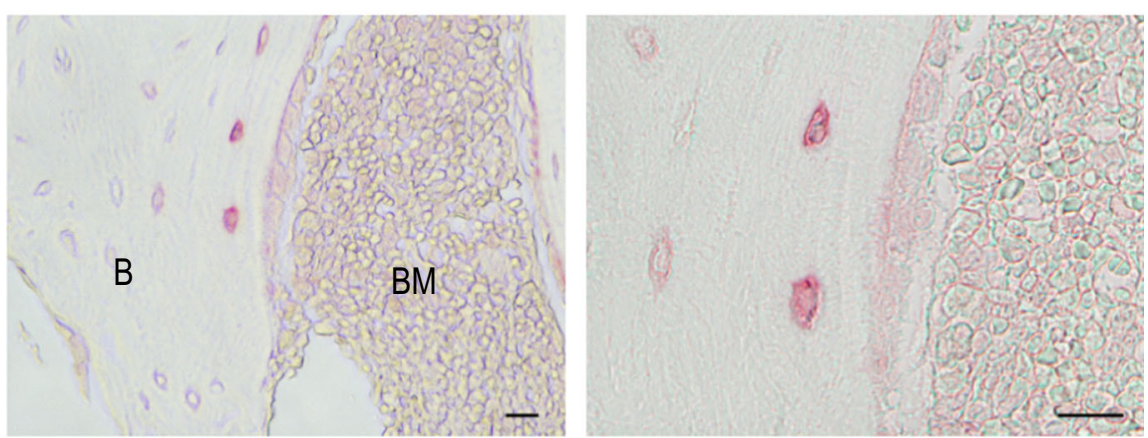

osteoblasts and partially upregulated osteoclast formation in bone marrow cultures. Although A7 antigen is a cell surface molecule, its ligand is unidentified. If A7 MAb acts as an agonist to the putative natural ligand, function of A7 antigen could be moderate upregulation of osteoclastogenesis with severe suppression of calcification (Fig. 11 upper panel). This event may occur in the early stage of bone remodeling, in which osteoclasts are recruited and initiates bone resorption at sites of bone remodeling. In contrast, if $\mathrm{A} 7 \mathrm{MAb}$ acts as an antagonist, the function of A7 antigen may be moderate inhibition of osteoclastogenesis with a marked induction of calcification (Fig. 11 lower panel). Such an event would be observed in the late stage of bone remodeling at the reverse phase, in which osteoblasts initiate new bone formation without recruiting osteoclasts. Such a regulatory molecule would be a key molecule in the molecular modulation of bone remodeling.

In the current study we demonstrated typical cell-surface localization pattern of A7 antigen in osteoblastic cell line ROS17/2.8 cells as well as bone marrow stromal cells with characteristics of osteoblasts [28, 29]. Extensive expression of A7 antigen at areas of higher cell density may be related to the up-regulation of the antigen expression in response to increased cell-cell adhesion or progression of cell maturation at these areas. In bone tissue, cuboidal cells covering the bone surface showed specific expression of A7 antigen in a typical membrane expression pattern. The colocalization of A7 antigen-expressing cells with ALPexpressing cells in bone tissue indicates that A7 antigenexpressing cells are osteoblasts, as expression of ALP is a marker of osteoblasts [30]. Because $100 \%$ of bone surface A7 antigen-positive cells expressed ALP while not all ALPpositive osteoblasts expressed A7 antigen, presence of A7 antigen-negative osteoblasts would indicate the presence of a distinct subpopulation of osteoblasts.

The membrane staining pattern of A7 antigen is similar to that of the cell adhesion molecule cadherin. It is known that osteoblasts express unique cell adhesion molecules known as OB cadherins (cadherin-11) [31, 32] which are also expressed in rat osteoblastic cell lines, ROS17/2.8 and UMR106 cells and in murine osteoblastic cell line MC3T3- 
a

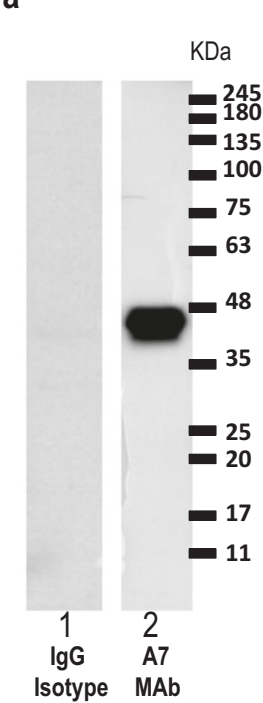

b

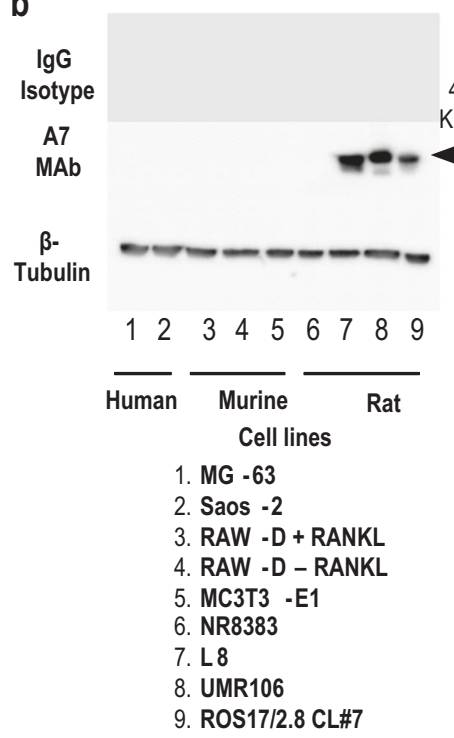

C

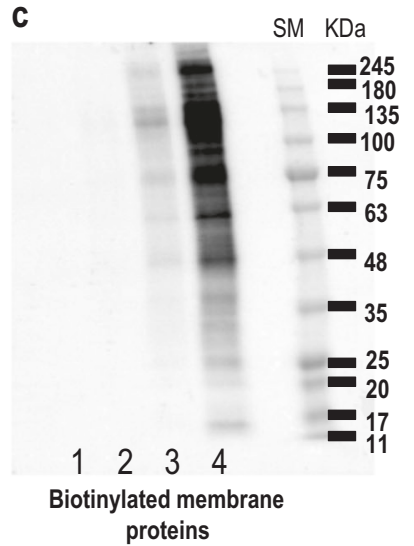

\section{d}

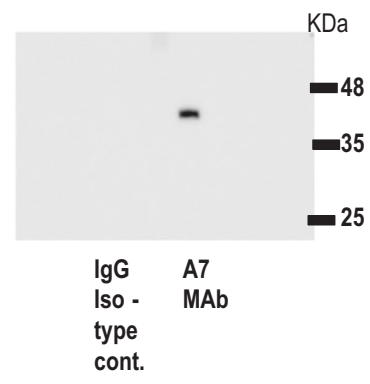

e

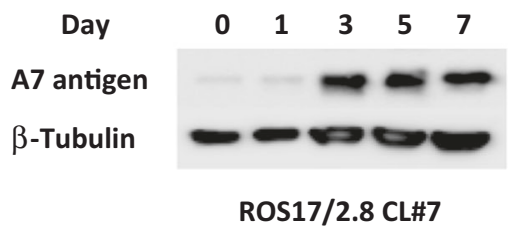

E1 cells [33]. Furthermore, the membrane expression pattern of cadherin-11 is similar to that of A7 antigen, in which intense immunoreactivity is observed at the marginal region of each osteoblast [34]. Cadherin-11 functions as the cell adhesion molecule through homophilic interaction of cadherin-11 molecule themselves in cadherin-11-expressing cells [35]. Molecular weight of cadherin-11 is $116 \mathrm{KDa}$ $[31,32]$ which is completely different from that of A7 antigen. A coordinative expression mechanism between differentiation stage, up-regulated adhesion through cadherin-11 and eliciting a maturation signal activating ALP expression has been reported [33]. Cell density-dependent up-regulation of cadherin-11 is similar to that of A7 antigen.

The marked expression of A7 antigen at areas of high cell density was also observed in bone marrow cultures for

Fig. 8 a Lineage and species specificity of the cell-surface A7 antigen expression: Western blotting and immunoprecipitation. Western blot analysis: Proteins extracted from cultured ROS17/2.8 CL\#7 cells (day 4) were subjected to $10 \%$ SDS-PAGE and Western blotting was performed as described in Materials and Methods. Following electroblotting, membrane was incubated with isotype control IgG (lane 1) or A7 MAb (lane 2), respectively. Although no band was detected by the isotype control $\mathrm{IgG}$, single protein band of $45 \mathrm{KDa}$ was clearly detected by A7 MAb. b Lineage and species specificity of A7 antigen expression: Western blot analysis of cell lysates extracted from cell lines of several species were subjected to $10 \%$ SDS-PAGE. Following electroblotting, membrane was incubated with isotype control $\mathrm{IgG}$ (upper panel), A7 MAb (middle panel) or anti- $\beta$-tubulin antibody (lower panel). Rat cell lines: Osteoblastic ROS17/2.8 CL\#7 cells, osteoblastic UMR106 cells, myoblast L8 cells and alveolar macrophage NR8383 cells; Murine cell lines: Osteoblastic MC3T3-E1 cells, osteoclast precursor RAW-D cells \pm RANKL $(50 \mathrm{ng} / \mathrm{ml})$ and human cell lines: Osteosarcoma Saos-2 cells and MG-63 cells. c Biotinylation of cell-surface proteins in ROS17/2.8 cell clone. Cell-surface proteins of ROS17/2.8 CL\#7 cells were labeled with biotin as described in Materials and Methods. Different dilutions of cell lysates involving biotinylated proteins were subjected to $10 \%$ SDS-PAGE and Western blotting followed by detection with Avidin HRP. Cell lysates were diluted as follows: Lane 1: 1/10000, Lane 2: 1/1000, Lane 3: 1/100, Lane 4: 1/10. d Immunoprecipitation of A7 antigen. Lysates of biotinylated cell-surface proteins of ROS17/2.8 CL \#7 cells were subjected to immunoprecipitation using isotype control $\mathrm{IgG}$ or $\mathrm{A} 7 \mathrm{MAb}$ as described in Materials and Methods. Eluted proteins were subjected to $10 \%$ SDS-PAGE followed by transfer to PVDF membrane and detected using Avidin HRP. No band was detected in the isotype control IgG lane. A7 MAb precipitated a cell surface antigen of molecular weight about $45 \mathrm{KDa}$. Data represent typical results among three independent experiments. e Induction time-course of A7 antigen expression in osteoblastic cells. ROS17/2.8 cells were seeded in 60 $\mathrm{mm}$ culture dishes. Cell lysates were prepared at $0,1,3,5$ and 7 days of culture. These cell lysates were subjected to quantitative western blot analysis. Time course blots were detected A7 antigen with A7 $\mathrm{MAb}$ (upper panel) and $\beta$-tubulin with anti- $\beta$-tubulin antibody (lower panel). Time-dependent induction of A7 antigen expression was apparent while expression level of $\beta$-tubulin did not change

forming osteoclasts. Our observation suggest that A7 antigen expression may be up-regulated when cells exit the cell cycle and start differentiation and maturation, and that cells at areas of lower cell density are still in the phase of proliferation. In staining of unfixed osteoblasts, we have shown a clear marginal staining representing a typical membrane distribution pattern of A7 antigen at various cell heights (z-axis). However, a dot-like or granular pattern was also observed at the basal and apical parts of some cells. In general, cell-surface receptors are internalized by endocytosis when they bind to their specific ligands [36]. As we had stained unfixed cells, the dot-like pattern may be related to antigen internalization.

In culture conditions that support osteoclastogenesis, expression of A7 antigen was specifically detected in osteoblast-like bone marrow stromal cells [28], while no expression of A7 antigen was detected in multinucleated osteoclasts in vitro, suggesting that A7 antigen expression is quite specific to cells of the mesenchymal origin. In contrast, osteoclast-specific cell surface antigen Kat1 antigen 


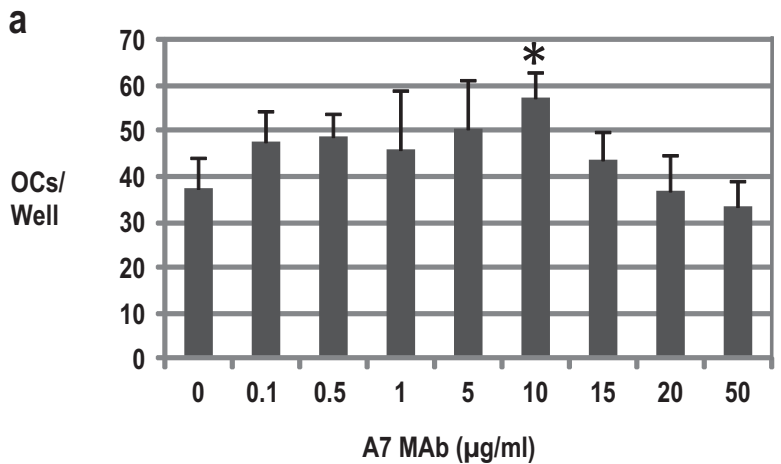

b
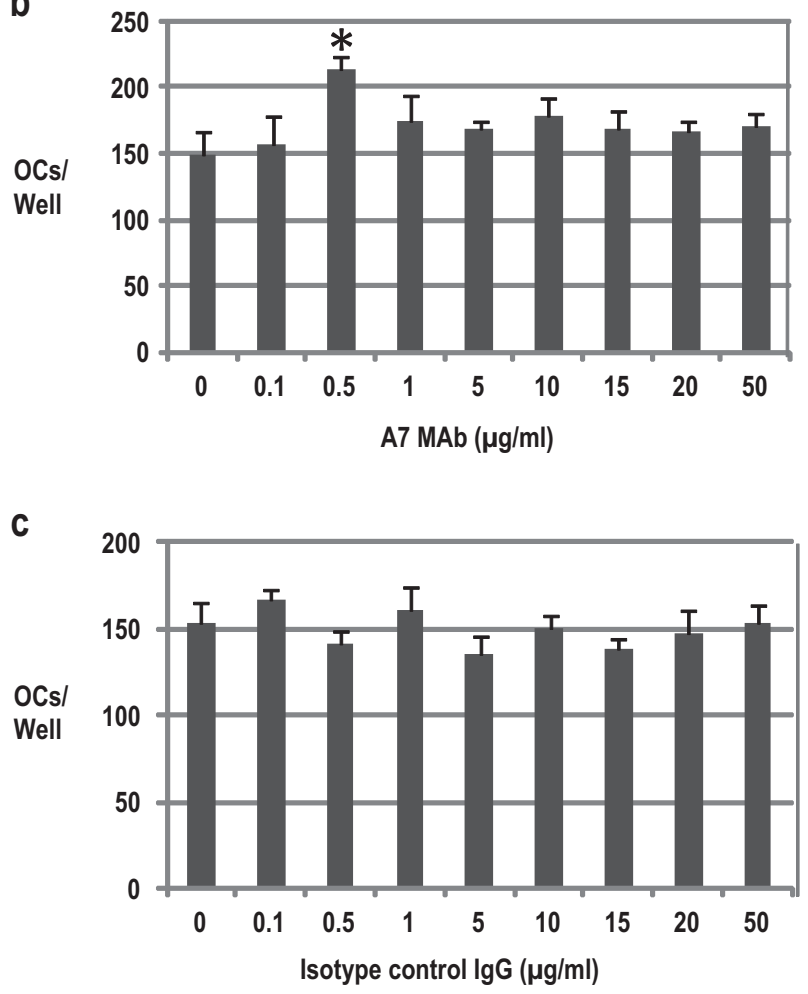

Fig. 9 Cross-linking of A7 antigen stimulates osteoclastogenesis Bone marrow cells of 4-week-old SD male rats were cultured in 96 multiwell plates $\left(0.2 \times 10^{6}\right.$ cells/well $)$ to induce osteoclasts as described in Materials and Methods (hybridoma screening) in the presence of various concentrations of A7 MAb or isotype control IgG. Cells were fixed and stained for TRAP at day 4 of culture. TRAP-positive cells having more than three nuclei were counted as osteoclasts. a Significant enhancement of osteoclastogenesis by A7 MAb in cultures of moderate osteoclast formation. b Significant up-regulation of osteoclastogenesis by A7 MAb in cultures of abundant osteoclast formation. Data represent mean \pm SEM $(n=6)$. c Absence of any modulatory effects on osteoclastogenesis by isotype control IgG1. ${ }^{*} p<0.05$, significant if compared to the control cultures containing no antibody

$[22,25-27]$ was not expressed in these osteoblast-like bone marrow stromal cells in culture. Completely different immunoreactivity of A7 MAb and Kat1 MAb may provide powerful tools to investigate precise interactions between osteoblasts and osteoclasts in vitro as well as in vivo.
Immunoblotting analysis clearly showed that A7 antigen was detected in rat osteoblastic cell lines ROS17/2.8 cells and UMR106 cells. A7 antigen was also detected in the myoblast cell line L8 derived from hind limb myoblasts of neonatal rats [37]. Myoblasts are derived from mesenchymal-derived precursors [38]. It has been reported that $\mathrm{C} 2 \mathrm{C} 12$ myoblasts are reprogrammed into mature osteoblasts by the stimulation with bone morphogenetic proteins (BMPs) as BMP-2 and BMP-4 [39, 40]. The common mesenchymal origin of L8 with the former cell lines possibly explains the detection of A7 antigen in L8 cells. A7 antigen may also have a regulatory function in the determination of the fate of the common differentiation unit into osteoblasts or into myotubes. Thus, this antigen may play a role in locomotive syndrome [41]. In contrast, A7 antigen was not expressed in rat alveolar macrophage cell line NR8383 [42]. These observations also confirm the specificity of A7 antigen expression in cells in the mesenchymal lineage not in cells in the haematopoietic lineage. Although the cell lines, murine MC3T3-E1 cells, human Saos-2 cells and human MG-63 cells, are osteoblastic and mesenchymal-derived cells, A7 antigen was not detected by A7 MAb in immunoblotting. This observation strongly suggests a stringent species specificity of A7 MAb, which is quite limited to rat species. Approximate molecular size of A7 antigen detected in immunoblotting was $45 \mathrm{KDa}$ and the size of the biotinylated membrane protein immunoprecipitated by A7 MAb was the same molecular size. These data strongly suggest that A7 antigen does not form any complex with other cell surface molecules expressed in osteoblasts.

In the current study, we describe the expression of A7 antigen in bone tissue. A7 antigen was expressed in a subset of cuboidal-shaped osteoblast-like cells located at the bone surface both in the metaphyseal trabecular bone and in surface periosteal cells of diaphyseal cortical bone. In addition, osteocytes present near the bone surface expressed A7 antigen. Wetterwald et al. [43] reported a transmembrane protein E11(30 KDa) specifically expressed in rat osteoblast-lineage cells. In bone tissue, E11 was expressed in cuboidal osteoblasts with polarized labeling toward the osteoid and uniform labeling in flattened-shaped osteoblasts. They also observed the antigen expression in early osteocytic cells and their processes near the bone surface. It was reported that E11 antigen may be signaling molecule in the process of osteoblast-osteocyte transition (osteocytogenesis) [44]. On the basis of immunohistochemical staining pattern of bone tissue, E11 antigen bears a similarity to A7 antigen in terms of staining of osteoblastic cells and osteocytes close to the bone surface, however A7 antigen was uniformly localized on cell-surface of osteoblasts and osteocytes without any polarized pattern. Moreover, A7 antigen was not clearly detected in cellular processes of 
a
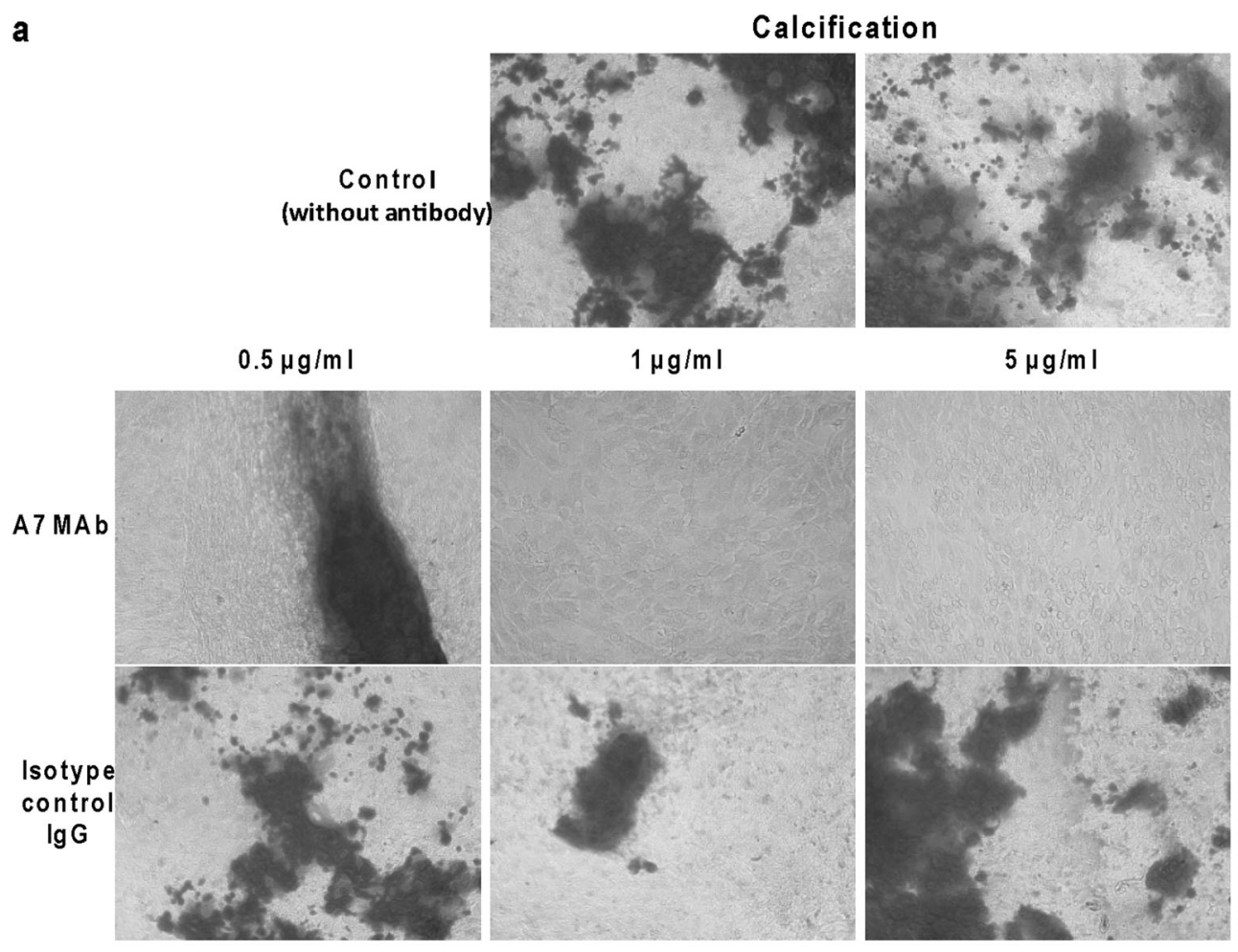

$1 \mu \mathrm{g} / \mathrm{m} \mathrm{I}$

$5 \mu \mathrm{g} / \mathrm{m} \mathrm{I}$

$10 \mu \mathrm{g} / \mathrm{m} \mathrm{I}$

b
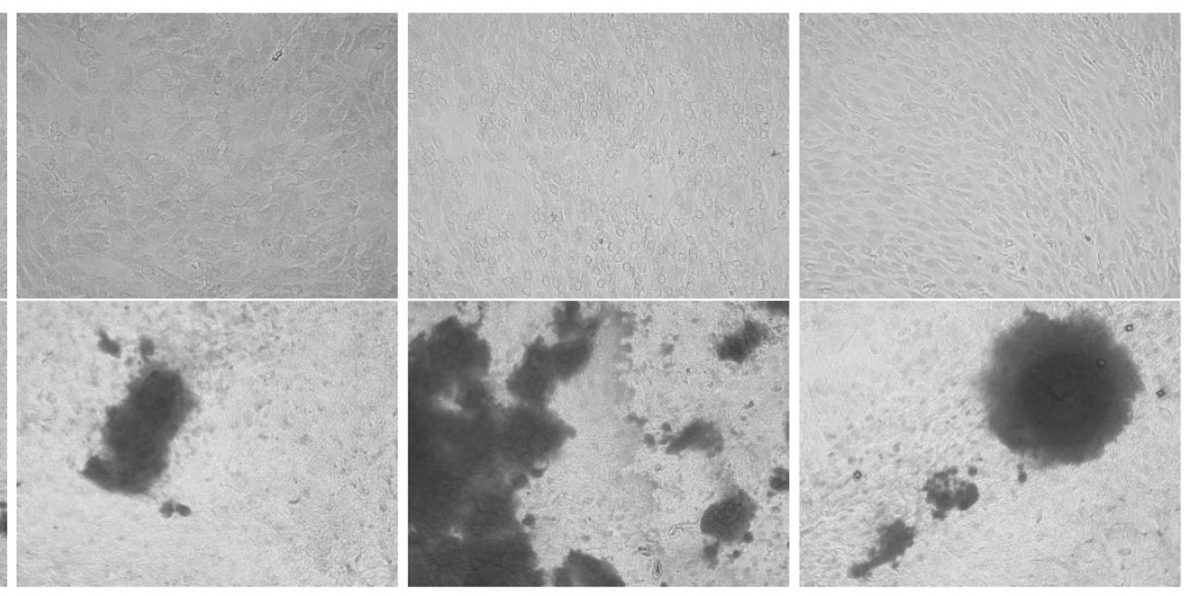

Proliferation (MTT Assay)

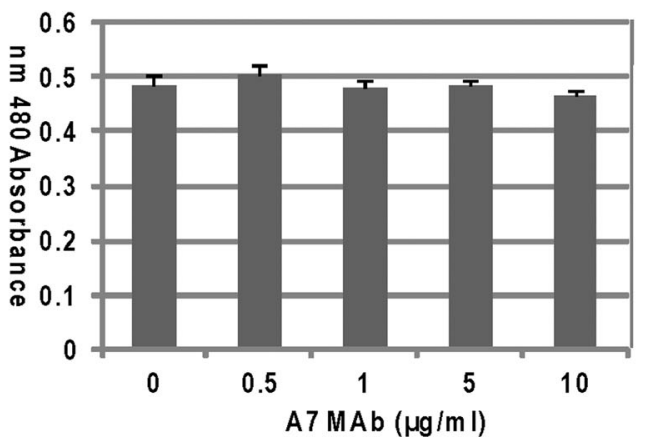

Fig. 10 Marked suppression of osteoblast-mediated calcification by cross-linking of A7 antigen without affecting cell proliferation Primary calvarial osteoblasts were cultured in 96 multi-well plates $\left(3.2 \times 10^{3}\right.$ cells/well) as described in Materials and Methods. a A7 MAb markedly inhibited calcification mediated by primary osteoblasts. Primary osteoblasts were cultured in calcification medium as described in Materials and Methods in the absence (upper panels) or in the presence of indicated concentrations of A7 MAb (middle panels) or isotype control IgG (lower panels). At day 21, cells were fixed and stained

osteocytes. These immunostaining data would further confirm that A7 antigen is different from E11 protein in addition to the difference in the molecular size. In respect to the cell adhesion molecules, Kawaguchi et al. [32] reported the expression of the transmembrane cadherin- 11 in the outer periosteum of calvaria, periosteal surface of femoral diaphysis and primary spongiosum in femoral trabecular bone with lower expression in the endosteal surface of femoral diaphysis, however they have shown no expression of

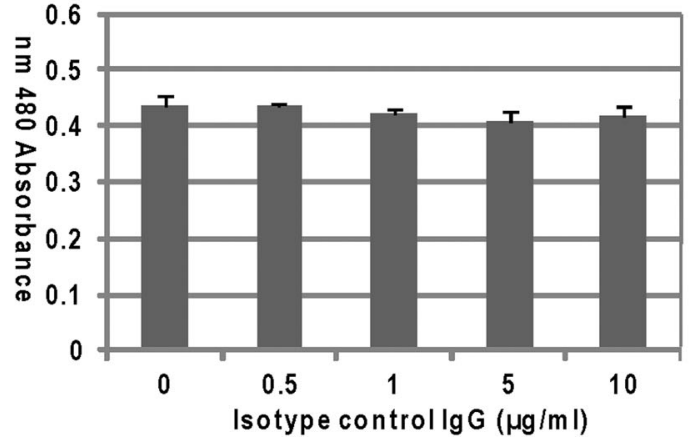

using alizarin red for the assessment of calcified deposits formation. $\mathbf{b}$ A7 MAb has no effect on cell proliferation and viability of primary osteoblasts. Calvarial osteoblasts were cultured in $\alpha$-MEM, $10 \%$ FBS in the presence of indicated concentrations of A7 MAb (left panel) or isotype control $\mathrm{IgG}$ (right panel). MTT proliferation assay was performed at day 4 culture. Data represent mean \pm SEM $(n=4)$. Experiments were performed two times and obtained almost identical results

cadherin-11 in osteocytes. In respect to the function of cadherin-11, the authors also demonstrated reduced calcification in calvarial cells obtained from cadherin-11 mutant mice, suggesting that the adhesive properties of cadherin-11 enhance the late stage of osteoblast differentiation. Furthermore, as the molecular size of cadherin-11 is different from that of A7 antigen, A7 antigen is not cadherine- 11 .

If $\mathrm{A} 7 \mathrm{MAb}$ has antagonistic activity to the putative natural ligand, the function of A7 antigen may be positive 


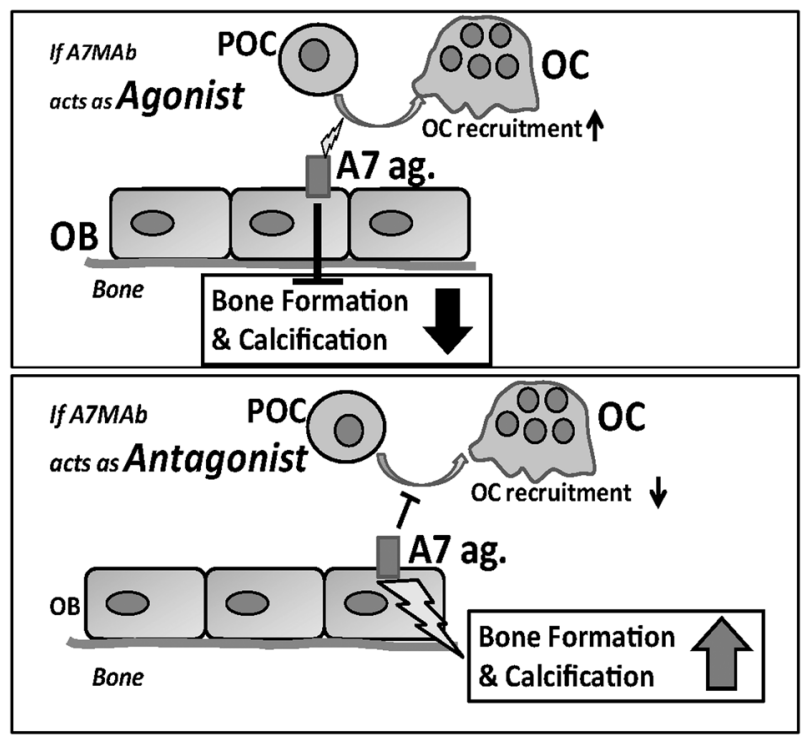

Fig. 11 Hypothetical model of the possible role of A7 antigen in the regulation of bone remodeling. If the function of $\mathrm{A} 7 \mathrm{MAb}$ is agonistic (Upper panel), expected function of A7 antigen may be as follows; Moderate up-regulation of osteoclast recruitment with marked suppression of bone formation and calcification. Such functions are thought to be required in the early stage of bone remodeling. In contrast, if the function of A7 MAb is antagonistic (Lower panel), function of A7 antigen would be as follows; Moderate inhibition of osteoclast recruitment with marked induction of bone formation and calcification. These events could be important in the late stage of bone remodeling. A7 ag., A7 antigen; A7 MAb, Anti-A7 monoclonal antibody; $O B$ osteoblast, $P O C$ preosteoclast, $O C$ osteoclast

regulation of osteoblast differentiation and maturation (Fig. 10). Eph-B4 expressed on cell surface of osteoblasts is a positive regulator of osteoblast differentiation in response to its ligand ephrin B2 expressed on cell surface of osteoclasts. Activation of osteoblast Eph-B4 by osteoclast ephrin B2 is referred to as "forward signaling" promoting osteoblast differentiation, whereas the action of EphB4 on ephB2 to suppress osteoclastogenesis is referred to as "reverse signaling" [16]. The possible function of A7 antigen is similar to that of EphB4 expressed on osteoblasts, however molecular size of EphB4 is $120 \mathrm{KDa}$ [45]. In primary calvarial-derived osteoblasts, blockade of EphB4/ephrin B2 pathway suppressed late osteoblast markers (osteocalcin, DMP and MEPE) and inhibited the deposition of calcified nodules without affecting proliferation or early differentiation markers [46], suggesting a role of EphB4/ephB2 axis in late stages of osteoblast differentiation especially in the step of osteoblast-osteocyte transition [47].

If A7 MAb exhibits agonistic activity, similar to that of a natural ligand, A7 antigen would act as a strong negative regulator of calcification with a slight ability to recruit osteoclasts (Fig. 10). In such a situation, A7 antigen would be an important regulator at early stages of bone remodeling. In contrast, if A7 MAb expressed antagonistic activity against the putative A7 antigen-natural ligand, A7 antigen may act as a powerful inducer of calcification with mild suppression of osteoclastogenesis. Such modulation may be required in the late stages of bone remodeling (Fig. 10). In this case, function of A7 antigen is similar to Eph-B4 expressed on cell surface of osteoblasts. Eph-B4 expressed in osteoblasts is reported to act as a positive regulator of osteoblast maturation in the reversal phase of bone remodeling [16]. The A7 antigen is not Eph-B4 (120 KDa) [45], but may have similar roles or cooperative roles in the molecular regulation of bone remodeling. In additiion, the expression of A7 antigen in surface cuboidal or flattened-shaped surface osteoblastic cells and osteocytes near the bone surface collectively suggest a possible role of A7 antigen in the reversal phase of bone remodeling where osteoclastic bone resorption is inhibited and mature osteoblasts start deposition of calcified bone matrices and subsequently converted into osteocytes.

Although the molecular entity of A7 antigen is not yet elucidated, this antigen may contribute to better understanding of the precise molecular events and signaling that occurs at the bone remodeling sites. Involvement of A7 antigen in the regulation of calcification mediated by mature osteoblasts would provide novel target for the treatment of bone diseases. As A7 antigen may be one inhibitory molecule acting directly on osteoclast precursors and suppressing bone resorption. It is also important to elucidate its bioactivity in vivo and in different pathological conditions of bone such as osteoporosis. The possible potential osteo-inductive or osteo-suppressive capacity of A7 antigen may be useful in the development of novel therapeutic approaches in bone diseases.

Acknowledgements The authors are grateful for the research grant from the Ministry of Education, Culture, Sports, Science and Technology of Japan. This research was supported by a Grant-in-Aid for Scientific Research, Challenging Research (15K15677).

\section{Compliance with ethical standards}

Conflict of interest The authors declare that they have no conflict of interest.

Statement of ethics All animal experiments were performed according to the guidelines for care and use of animals of Kyushu University.

Publisher's note: Springer Nature remains neutral with regard to jurisdictional claims in published maps and institutional affiliations.

\section{References}

1. Feng X, McDonald JM. Disorders of bone remodeling. Annu Rev Pathol. 2011;6:121-45.

2. Teitelbaum SL. Bone resorption by osteoclasts. Science. 2000;289:1504-8.

3. Parfitt AM. Osteonal and hemi-osteonal remodeling: the spatial and temporal framework for signal traffic in adult human bone. $\mathbf{J}$ Cell Biochem. 1994;55:273-86. 
4. Parfitt AM. Skeletal heterogeneity and the purposes of bone remodeling: implications for the understanding of osteoporosis. (Marcus R, Feldman D, Kelsey J. eds.) In: Osteoporosis 4th ed. 2013. p. 855-72.

5. Martin TJ, Seeman E. New mechanisms and targets in the treatment of bone fragility. Clin Sci. 2007;112:77-91.

6. Hienz SA, Paliwal S, Ivanovski S. Mechanisms of bone resorption in periodontitis. J Immunol Res. 2015;6:15486.

7. Zhang A, Wang P, Ma X, et al. Mechanisms that lead to the regulation of NLRP3 inflammasome expression and activation in human dental pulp fibroblasts. Mol Immunol. 2015;66:253-62.

8. Schett G, Gravallese E. Bone erosion in rheumatoid arthritis: mechanisms, diagnosis and treatment. Nat Rev Rheumatol. 2012;8:656-64.

9. Shiratori T, Kyumoto-Nakamura Y, Kukita A, et al. IL-1 $\beta$ induces pathologically activated osteoclasts bearing extremely high levels of resorbing activity: a possible pathological subpopulation of osteoclasts, accompanied by suppressed expression of Kindlin-3 and Talin-1. J Immunol. 2018;200:218-28.

10. Lacey DL, Timms E, Tan HL, et al. Osteoprotegerin ligand is a cytokine that regulates osteoclast differentiation and activation. Cell. 1998;93:165-76.

11. Yasuda H, Shima N, Nakagawa N, et al. Osteoclast differentiation factor is a ligand for osteoprotegerin/osteoclastogenesis-inhibitory factor and is identical to TRANCE/RANKL. Proc Natl Acad Sci USA. 1998;95:3597-602.

12. Fuller K, Wong B, Fox S, et al. TRANCE is necessary and sufficient for osteoblast-mediated activation of bone resorption in osteoclasts. J Exp Med. 1998;188:997-1001.

13. Boyle WJ, Simonet WS, Lacey DL. Osteoclast differentiation and activation. Nature. 2003;423:337-42.

14. Hofbauer LC, Khosla S, Dunstan CR, et al. The roles of osteoprotegerin and osteoprotegerin ligand in the paracrine regulation of bone resorption. J Bone Miner Res. 2000;15:2-12.

15. Wang HU, Chen ZF, Anderson DJ. Molecular distinction and angiogenic interaction between embryonic arteries and veins revealed by ephrin-B2 and its receptor Eph-B4. Cell. 1998;93:741-53.

16. Zhao C, Irie N, Takada $\mathrm{Y}$, et al. Bidirectional ephrinB2-EphB4 signaling controls bone homeostasis. Cell Metab. 2006;4: 111-21.

17. Kukita T, Wada N, Kukita A, et al. RANKL-induced DC-STAMP is essential for osteoclastogenesis. J Exp Med. 2004;200:941-6.

18. Watanabe T, Kukita T, Kukita A, et al. Direct stimulation of osteoclastogenesis by MIP-1alpha: evidence obtained from studies using RAW264 cell clone highly responsive to RANKL. J Endocrinol. 2004;180:193-201.

19. Rodan SB, Noda M, Wesolowski G, et al. Comparison of postreceptor effects of 1-34 human hypercalcemia factor and 1-34 human parathyroid hormone in rat osteosarcoma cells. J Clin Invest. 1988;81:924-7.

20. Kohler G, Milstein C. Continuous cultures of fused cells secreting antibody of predefined specificity. Nature. 1975;256:495-7.

21. Reading CL. Theory and methods for immunization in culture and monoclonal antibody production. J Immunol Meth. 1982;53:261-91.

22. Kukita T, Kukita A, Nagata K, et al. Novel cell-surface Ag expressed on rat osteoclasts regulating the function of the calcitonin receptor. J Immunol. 1994;153:5265-73.

23. Takano T, Li Y, Kukita A, et al. Mesenchymal stem cells markedly suppress inflammatory bone destruction in rats with adjuvantinduced arthritis. Lab Invest. 2014;94:286-96.

24. Kukita A, Kukita T, Hata K, et al. Heat-treated osteoblastic cell (ROS17/2.8)-conditioned medium induces the formation of osteoclast-like cells. Bone Miner. 1993;23:113-27.
25. Kukita T, Kukita A, Xu L, et al. Successful detection of active osteoclasts in situ by systemic administration of an osteoclastspecific monoclonal antibody. Calcif Tissue Int. 1998;63:148-53.

26. Kukita T, Kukita A, Watanabe T, et al. Osteoclast differentiation antigen, distinct from receptor activator of nuclear factor kappa B, is involved in osteoclastogenesis under calcitonin-regulated conditions. J Endocrinol. 2001;170:175-83.

27. Matsubara R, Kukita T, Ichigi $\mathrm{Y}$, et al. Characterization and identification of subpopulations of mononuclear preosteoclasts induced by TNF-alpha in combination with TGF-beta in rats. PLoS One. 2012;7:e47930.

28. Takahashi N, Udagawa N, Akatsu T, et al. Deficiency of osteoclasts in osteopetrotic mice is due to a defect in the local microenvironment provided by osteoblastic cells. Endocrinology. 1991;128:1792-6.

29. Kukita A, Kukita T, Shin JH, et al. Induction of mononuclear precursor cells with osteoclastic phenotypes in a rat bone marrow culture system depleted of stromal cells. Biochem Biophys Res Commun. 1993;196:1383-9.

30. Aubin JE. Regulation of osteoblast formation and function. Rev Endocr Metab Disord. 2001;2:81-94.

31. Marie PJ, Hay E, Modrowski D, et al. Cadherin-mediated cell-cell adhesion and signaling in the skeleton. Calcif Tissue Int. 2014;94:46-54.

32. Kawaguchi J, Azuma Y, Hoshi K, et al. Targeted disruption of cadherin-11 leads to a reduction in bone density in calvaria and long bone metaphyses. J Bone Miner Res. 2001;16:1265-71.

33. Okazaki M, Takeshita S, Kawai S, et al. Molecular cloning and characterization of OB-cadherin, a new member of cadherin family expressed in osteoblasts. J Biol Chem. 1994;269:12092-8.

34. Huang CF, Lira $\mathrm{C}$, Chu $\mathrm{K}$, et al. Cadherin-11 increases migration and invasion of prostate cancer cells and enhances their interaction with osteoblasts. Cancer Res. 2010;70:4580-9.

35. Takeichi M. Morphogenetic roles of classic cadherins. Curr Opin Cell Biol. 1995;7:619-27.

36. Lodish H, Berk A, Zipursky SL. et al. Receptor-mediated endocytosis and the sorting of internalized proteins. (Baltimore D ed.) In: Molecular Cell Biology. 4th ed. New York: W. H. Freeman; 2000.

37. Kaufman SJ, Lawless ML. Thiodigalactoside binding lectin and skeletal myogenesis. Differentiation. 1980;16:41-48.

38. Holtzer H. Myogenesis. In: Schjeide O, Devillis J, ed. In cell differentiation. New York: Van Nostrand-Reinhold; 1970. p. 476-503.

39. Katagiri T, Yamaguchi A, Komaki M, et al. Bone morphogenetic protein-2 converts the differentiation pathway of $\mathrm{C} 2 \mathrm{C} 12$ myoblasts into the osteoblast lineage. J Cell Biol. 1994; 127:1755-66.

40. Feng L, Cook B, Tsai SY, et al. Discovery of a small-molecule BMP sensitizer for human embryonic stem cell differentiation. Cell Rep. 2016;15:2063-75.

41. Nakamura K, Ogata T. Locomotive syndrome: definition and management. Clin Rev Bone Miner Metab. 2016;14:56-67.

42. Helmke RJ, Boyd RL, German VF, et al. From growth factor dependence to growth factor responsiveness: the genesis of an alveolar macrophage cell line. Vitr Cell Dev Biol. 1987;23:567-74.

43. Wetterwald A, Hofstetter W, Cecchini MG, et al. Characterization and cloning of the E11 antigen, a marker expressed by rat osteoblasts and osteocytes. Bone. 1996;18:125-32.

44. Hadjiargyrou M, Rightmire EP, Ando T, et al. The E11 osteoblastic lineage marker is differentially expressed during fracture healing. Bone. 2001;29:149-54.

45. Nguyen TM, Arthur A, Panagopoulos R, et al. EphB4 expressing stromal cells exhibit an enhanced capacity for hematopoietic stem cell maintenance. Stem Cells. 2015;33:2838-49. 
46. Allan EH, Hausler KD, Wei T, et al. EphrinB2 regulation by PTH and PTHrP revealed by molecular profiling in differentiating osteoblasts. J Bone Miner Res. 2008;23:1170-81.
47. Takyar FM, Tonna S, Ho PWM, et al. EphrinB2/EphB4 inhibition of osteoblast lineage modifies the anabolic response to parathyroid hormone. J Bone Miner Res. 2013;28:912-25. 